Journal of Engineering Sciences, Assiut University, Vol. 40 No 6 pp.1875-1894 November 2012

\title{
The Effect of the Third Generation of the Digital Revolution on Developing the Formulation of Contemporary Architectural Forms
}

\author{
Dr. Mohamed Wahba Ebrahim \\ Asst. Prof. at Architectural Engineering and Environmental Design Department \\ College of Engineering and Technology \\ Arab Academy for Science \& Technology \& Maritime Transport
}

(Received April 9, 2012 Accepted August 14, 2012)

\section{Abstract}

Since the earliest beginning of history, architectural fortresses have acquired varied forms and masses. It was possible to interpret justifications for using many of them; however, usage of some of these forms and masses is still considered as a perplexing mystery for scholars, analysts and interested persons in the topic of mass formulation in architecture. For example, the Giza Pyramids in Egypt which were built 4500 years ago, whose pyramidal mass, which was the output of several astronomic and mathematical relations in the pyramid form, the world is still till our present day analyzing the inherent ideas beyond them. Throughout ages, ideas of approaching architectural masses differed till the architectural masses of buildings at the end of the twentieth and the onset of the twenty-first centuries reflected several matters. The most important among these are the architectural, constructive and mechanical sciences and the high technological reality.

Thus, the architectural form has turned from static masses to dynamic masses during the digital and information revolution which concentrated on producing supplementary ideas and programs in design. In fact, design by the help of computer programs achieves the uniqueness and distinctiveness of ideas, and the inventiveness of the mass and its transformations so that ideas of the architect are freed away from the traditional limitations and restrictions. With the development of computer technology with the digital, then the information, revolution, numerous generations appeared : the first was the computer technology (computer programs), the second was the digital (internet) and the third was the information (Infomedia). This development has had its direct effect on architecture and urbanization. Architecture has had to correspond with modern needs for users; therefore, what was called digital architectural thinking or the third generation for creating architectural masses, and in turn the third generation of the contemporary urbanization texture, has emerged. This represents the research problem in what we do not find in our local architecture due to following the world in this technology because architects cannot identify it. Hence, it appears the importance of getting to identify what the architectural thought tools have reached and its passage through several generations so that it keeps updated with the age of technology and employs what technology provides of contribution in creating a building or a city that undergoes all kinds of tests before its implementation. All these ideas are interwoven is an architectural entity that contains them which represents a digital reflection on the urban design controlled by information by what is called urbanization and architecture of the third generation of the age of technology in the twentieth-first century. Hence, the research is divided into several axes. To begin with, the first axis deals with the 
historical background of the architectural mass influence by the industrial, then the digital and the information revolutions passing through the second axis which discusses the influence of the information revolution on the formation of buildings' masses by applying computer programs and identifying what the new methods could provide to the design process of creativity. Then comes the third axis which reflects the manner by which high buildings got influenced by digital technology. As for the fourth axis, it deals with information revolution and its realization of the third generation's dream in terms of technology cities. Finally, the research discusses testing the effect of these methods in the design process through conducting a questionnaire which concludes to an inference which is the information methods and techniques promotes the efficiency of the design process. The research recommends the necessity that students, academics, professionals and practitioners in the field of architectural engineering should identify the information elements and techniques in architectural design in order to correspond to the age development.

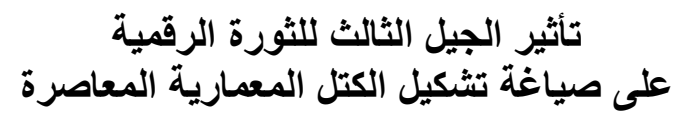

\section{أ.م.د. محمد وهبة إبراهيم}

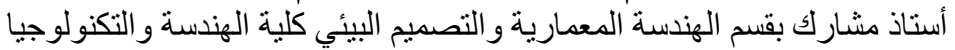

الأكاديمية العربية للعلوم و التكنولوجيا والنية النقل البحري بالإسكندرية النية والنية

منذ بدايات التاريخ اتخذت الصروح المعمارية أنكالا وكتلا متنو عة ، أمكن تفسبر مبررات استخدام الكثير

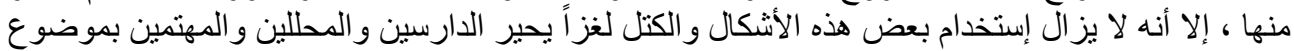

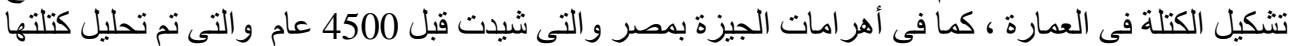

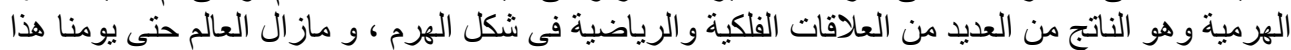
يقوم بتحليل الافكار الكامنة وراءها.

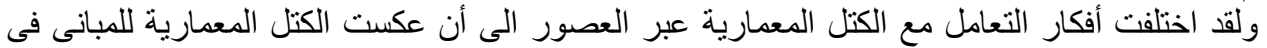

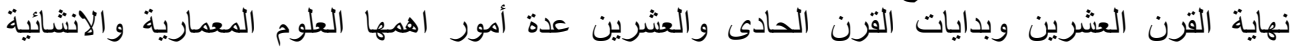

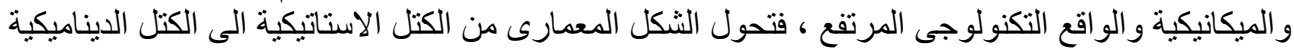

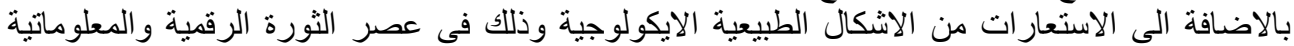

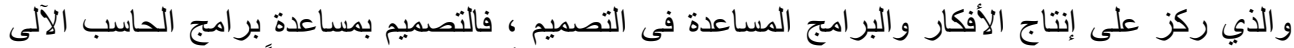

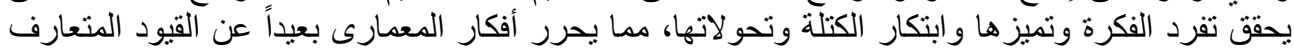

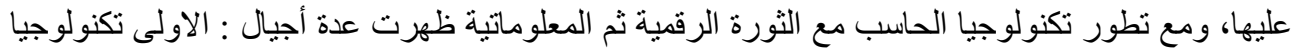

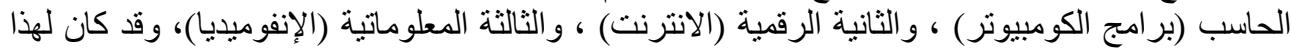

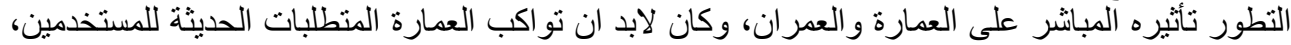

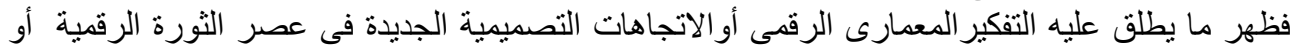

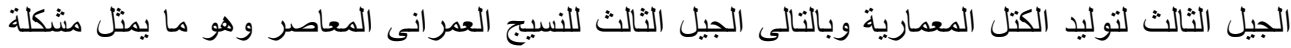

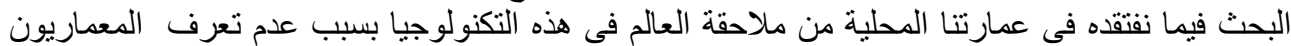
عليها.

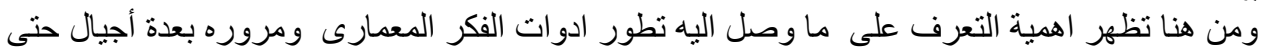

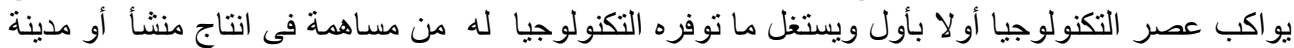

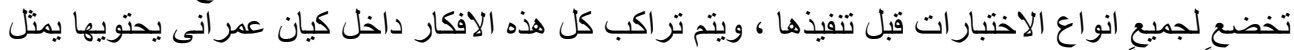

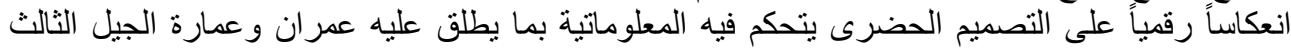

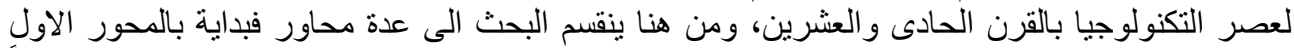
ويتتاول الخلفية التاريخية لتأثر الكتلة المعمارية بالثورات الصناعية ثم الرقمية ثم المعلوماتية ومروراً 
بالمحور الثانى والذى يتناول تأثير الثورة المعلوماتية على تشكيل كتل المبانى بإستخدام برامج الحاسب و

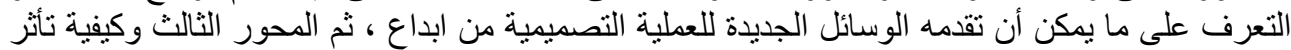

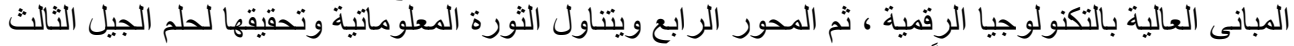

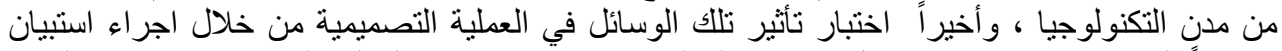

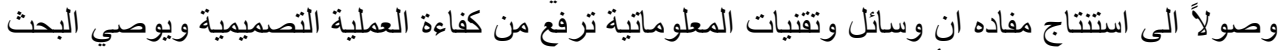

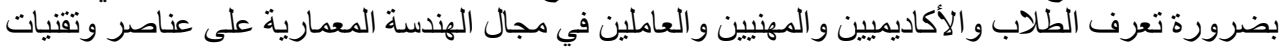
المعلوماتية في التصميم المعماري وذلكادين لمو اكبة تطور العصنر. إثكالية البحث

إن اغلب الدر اسات السابقة والتى تناولت المعلوماتية والعمارة ركزت على موضوع التطور التكنولوجي بشكل

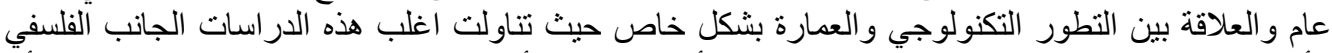

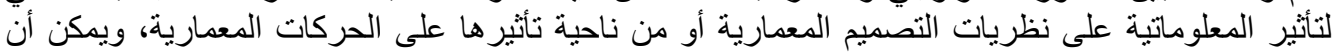

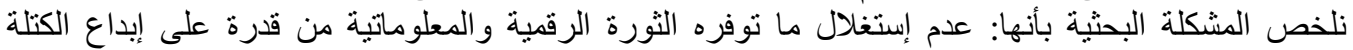

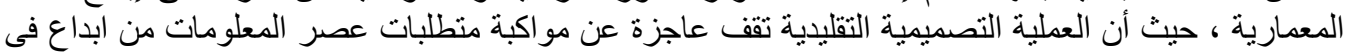

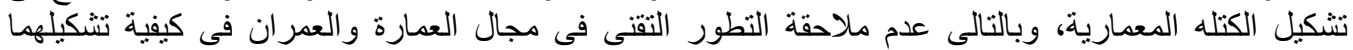

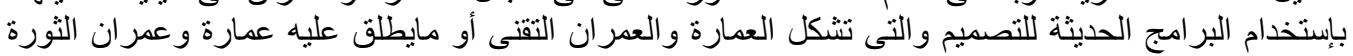
الرقمية و المعلوماتية وخاصة في عصر الجئية الجيل الثالث للتكنولوجيا.

هدف البحثة. البحث الى التعرف على كيفية تأثر العملية التصميمية بتطبيقات الثورة المعلوماتية سواء للمبانى

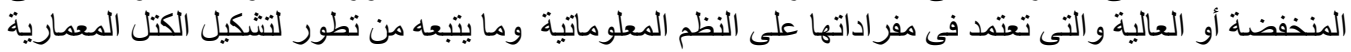

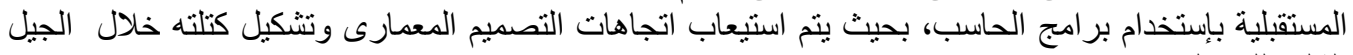

الثالث للتكنولوجيا.

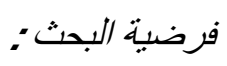

ويفترض البحث ان اعتماد النظم والتقنيات المعلوماتية في التصميم برفع من كفاءة العملية التصميمية وينمي

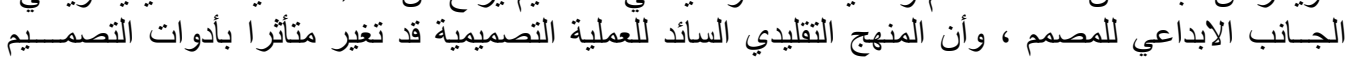

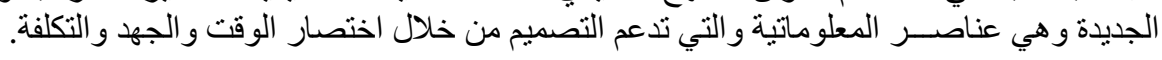

$$
\text { منهجية البحث: }
$$

$$
\text { للوصول الى هدف البحث يتم اتباع منهجين اساسيين هما : }
$$

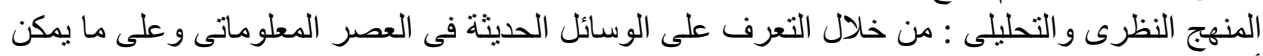

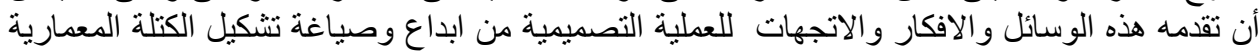

المعاصرة.

المنهج التطبيقى: التعرف على قدرة استخدام تطبيقات بر امج الحاسب فى نشكيل العمارة من خلال استبيان

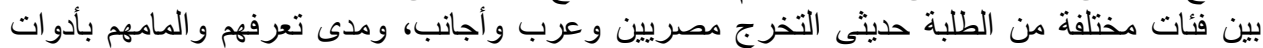

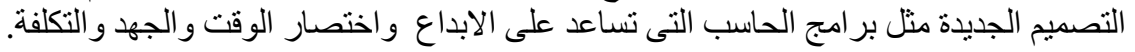

\section{1 - المحور الأول : الخلفية التاريخية لتثكيل الكتلة المعمارية}

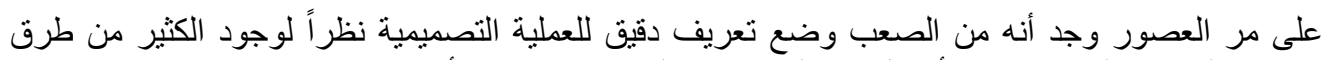

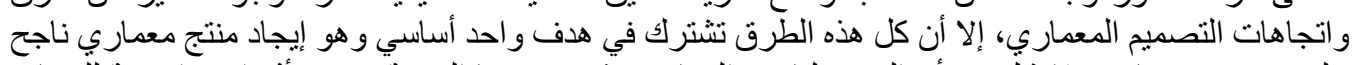

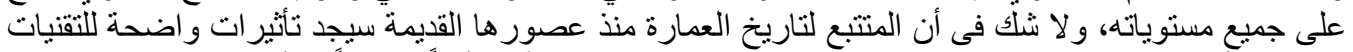

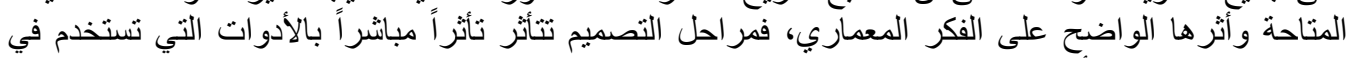

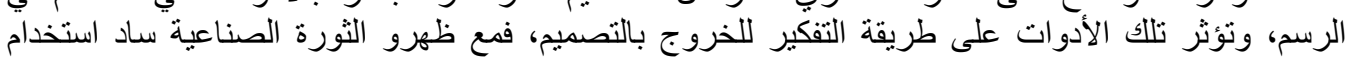


المساطر و المثلثات المستوية وساد الحل على شبكات مربعة و المكعبات في تشكيل الكتل المعمارية ، وكان الاتجاه

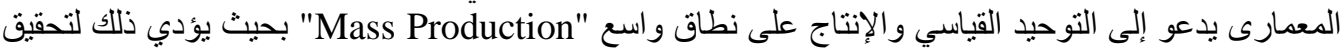

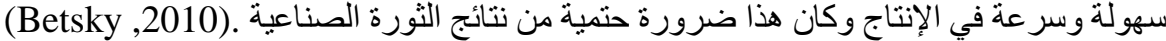

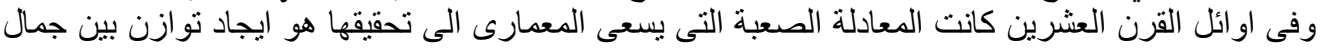

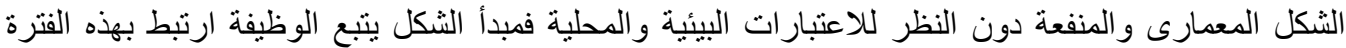
كعمارة الحداثة و اهتمامها بالاشكال الاستاتيكية الهندسية البسيطة. (Jencks, 2000)

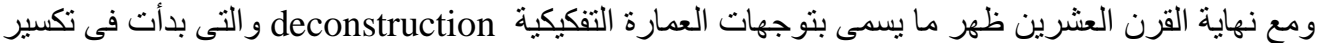

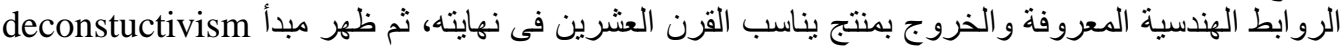

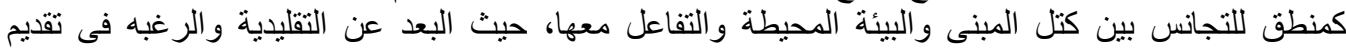

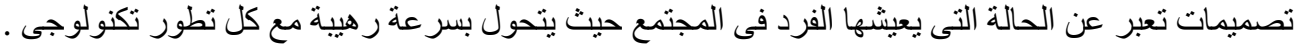

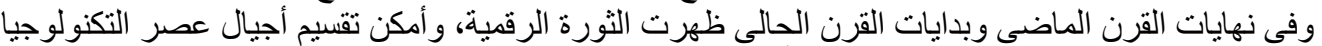

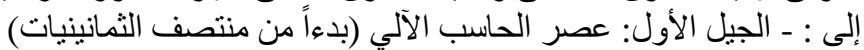

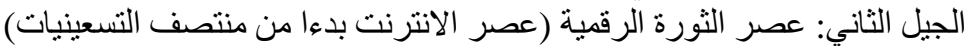

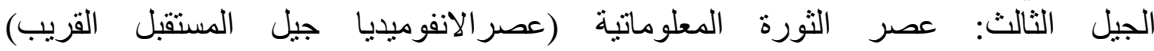

(Kolarevic,2011)

ولقد خضع التشكيل المعماري فى عصر الثورة الرقمية متمثلا فى جيلها الثالث وهو المعلوماتية لقواعد إضافية

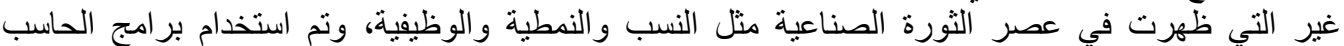

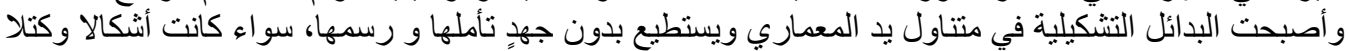

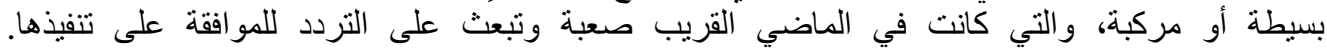
(Kolarevic,2001) وبظهور الثورة الرقمية نجدها قدمت دعوة كبيرة الى فهم و اعادة صياغة مفردات لغة التشكيل المعمارى وتأثيره

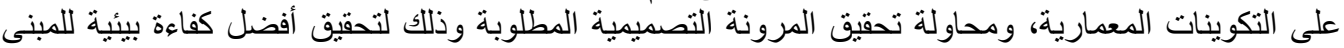

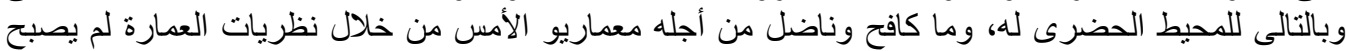

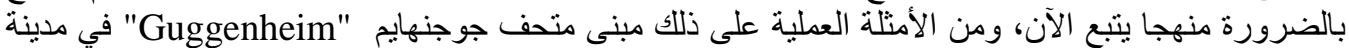

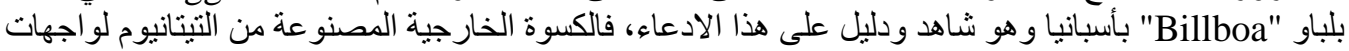

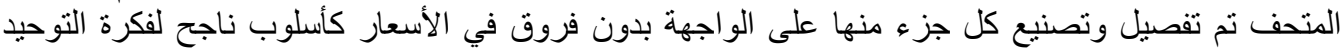

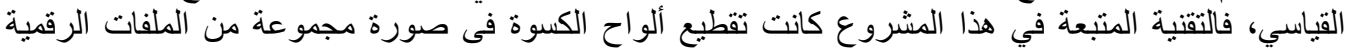

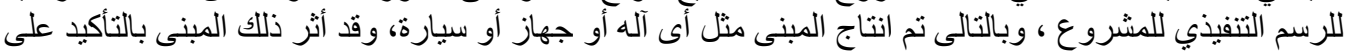

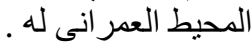

$$
\text { الاتجاهات التى تساعد الدعدارى لاستلهام فكرته قبل التعبير عنها بإِتخدام برامج الحاسب. }
$$

أ الاتجاه الدورفولوجى : (Morphological Approach)

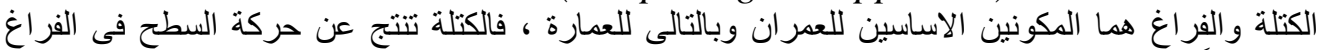

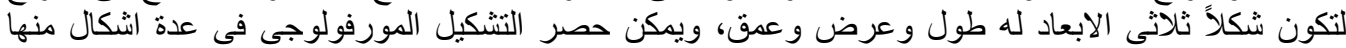
الرومانسى، و الوظيفى، و الآلى، و التعبيرى، و والعضوى (Kolarevic, 2000)

ب- الاتجاه الميثولوجى: (Mythological Approach)

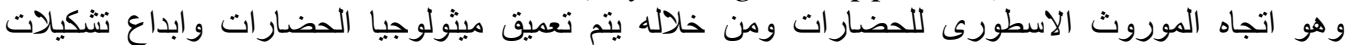

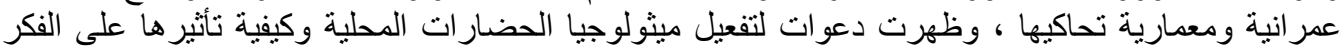

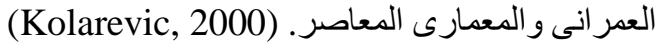

ج - الاتجاه الايكولوجى والحيوى : (U.N.C., (Ecological \& Biomimatic Approach) 1996)

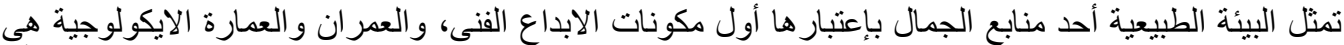

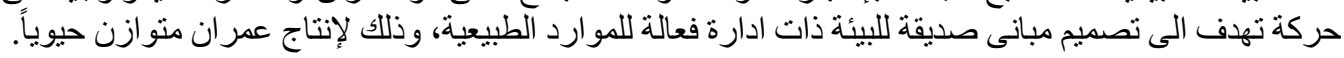




\section{لدـ الاتجاه البيئي : (Environmental Approach)}

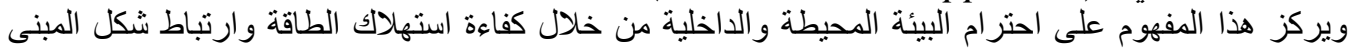

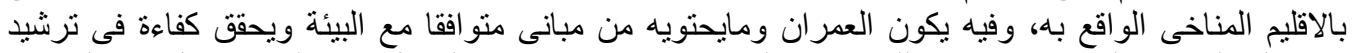

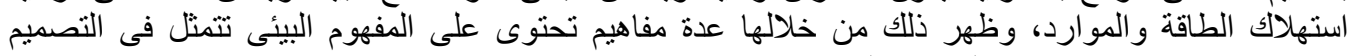

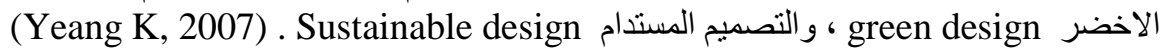

ولقد رأى المعماريون فى العصر الرقمى انهم فى تحد لقدر اتهم الابداعية وفى تناغم وفكر جديد لإخر اج تنشكيلات

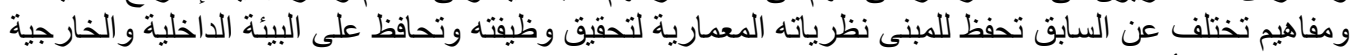

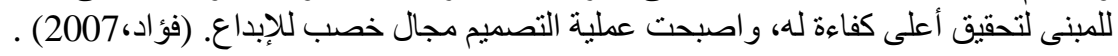

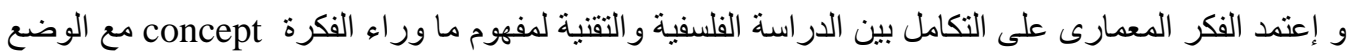

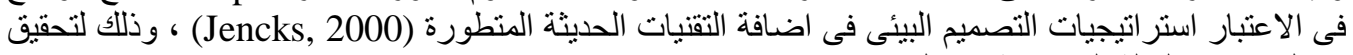

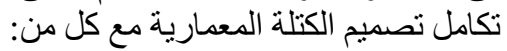
التفاعل الحيوى Metabolism : ويدرس متطلبات المشروع ويستخدم التكنولوجيا التى تلائم وتتفاعل وتكنولوجى.

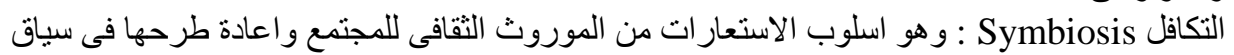

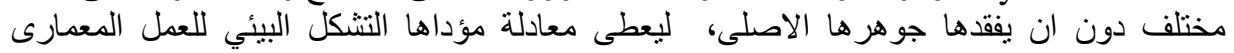

.Environmental form

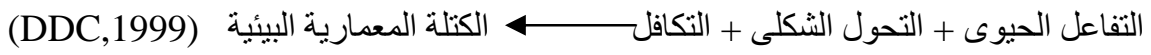

Metabolism + Metamorphosis + Symbiosis $\longrightarrow$ Environmental Form

وفى اطار موازى لهذا الاتجاه بدأ بعض المعماريين في استخدام برامج الحاسب الآلى بثكل أكبر في أعمالهم

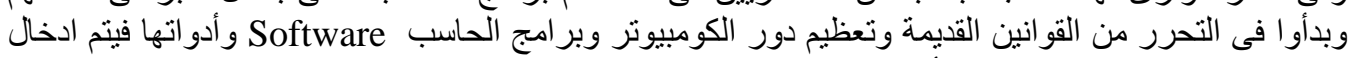

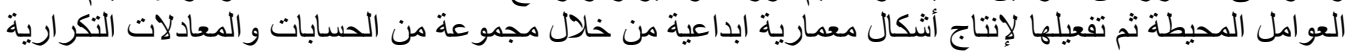

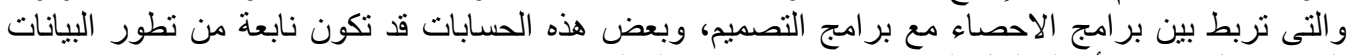

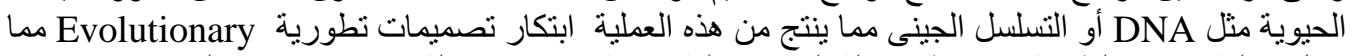

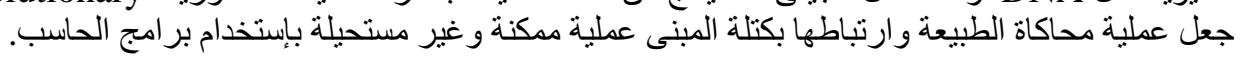

\section{2- المحور الثانى : دور الجيل الثالث للتكنولوجيا فى صياغة الكتلة المعمارية}

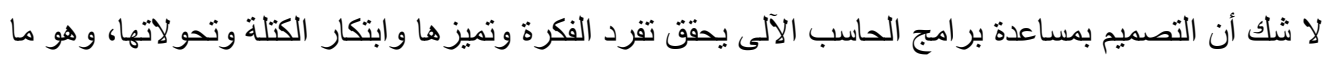

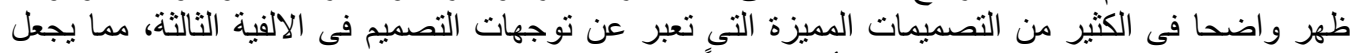

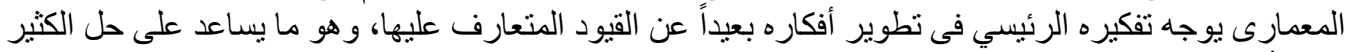

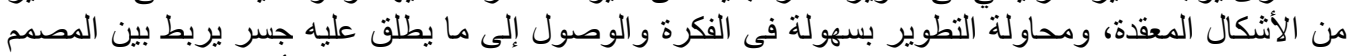

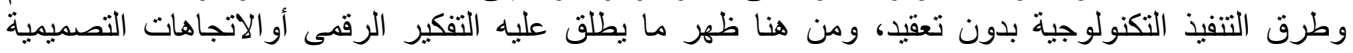

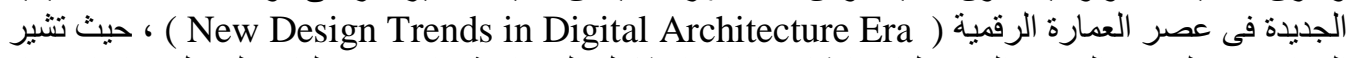

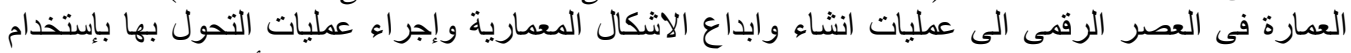

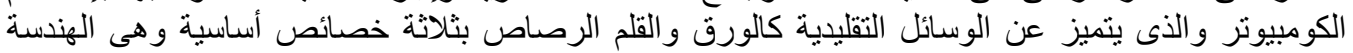

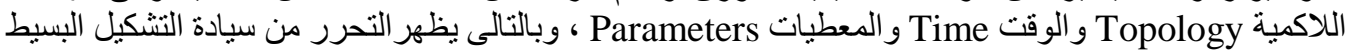
للفر اغات المعمارية. (Kolarevic,2011) وبناء على مفاهيم علوم الحاسب وبر الرير امج الرسم ثلاثى الابعاد أمكن تحديد تنكيل الكتل الحديثة فى العمارة خلال

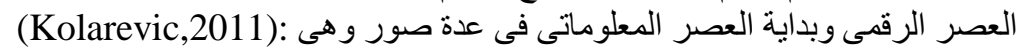


1- Performative architecture (Digital analysis)

2- Topological architecture (Topological space)

3- Isomorphic architecture (Isomorphic surfaces)

4- Animate architecture (Motion kinematics \& dynamics)

5- Metamorphic architecture (Key shape animation)

6- Parametric architecture (Parametric design)

7- Evolutionary architecture (Genetic algorithms)

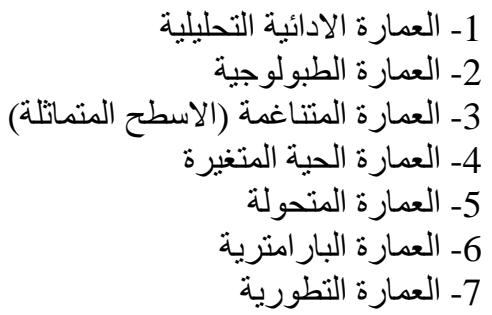

\section{2-1 Performative architecture (digital analysis)}

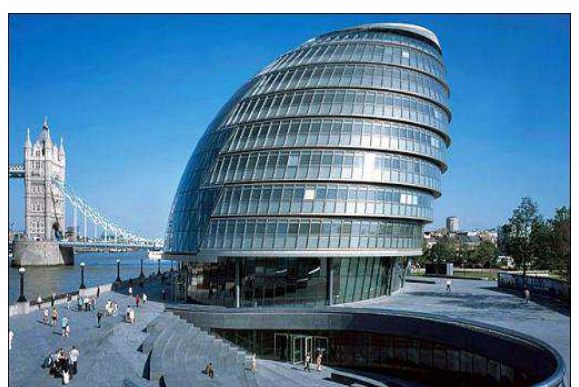

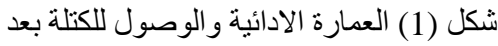
التحليل الرقمى للرياح وحركة الثمس.

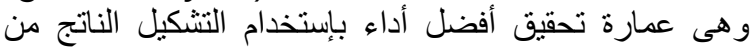

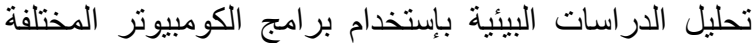

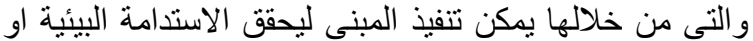

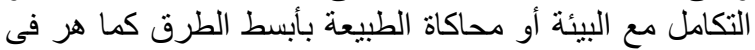

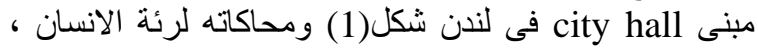
وهذا الاسلوب فى التصميم يعتمد على تصميم تفاصيل دقيقة للمعالجات البيئية (Kolarevic,2000) ، ولئية واستخدام مواد

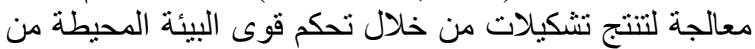
رياح وحركة شمس ودرجات حر ارة .... الخ.

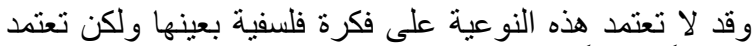

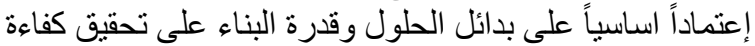

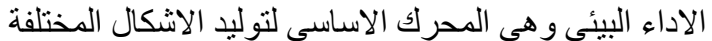

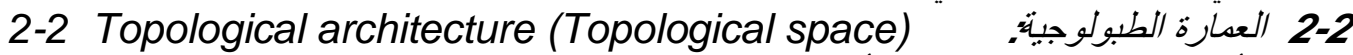

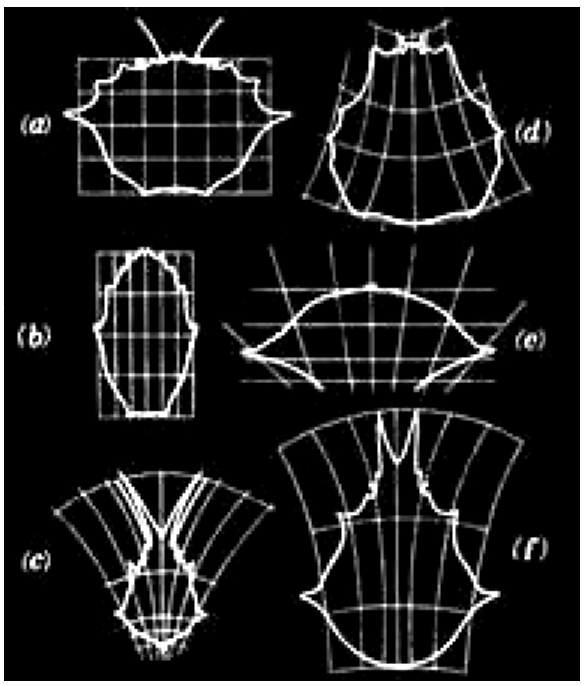

شكل (2) تحول الاشكال نتيجة التحكم فى الثبكات
إن شكل أى جسم هو عبارة عن خريطة أو شبكة لها عدة قوى فئ وبتحريك وشد هذه الثبكة فى اتجاهات مختلفة يعطى اشكالا مختلفة شكل(2)، وهو ما يطلق عليها العمارة الطبولوجية

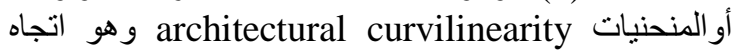
يبعد عن الاتجاه التفكيكى أو الهدم deconstruction الذى منى

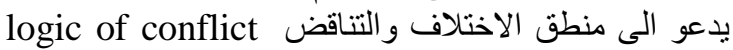
بينما بدعو هذا الاتجاه الى منطق and contradiction ترابط الكتلة يتبع التدفق وقد يكون تدفقا فى الطاقة الحيوية more fluid logic of connectivity بالمبنى كتمثيل لنظام ايكولوجي طبيعي من خلال نتبع تدفق

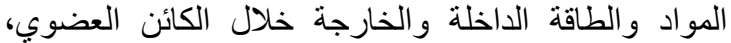

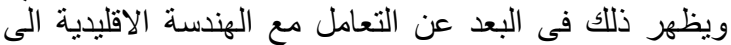

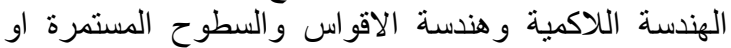
الاسطح المرنة rubber-sheet والتى تعرف فئ ولى علوم الرياضيات NURBS وهى اختصار الحروف الاولى لجملة ، (3on-Uniform Rational B-Splines) ويسهل التحكم بها وتعديلها من خلال التحكم فى قيم نقاطها

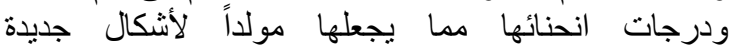

(4olarevic 2011) 
تأثير الجيل الثالث للثورة الرقمية
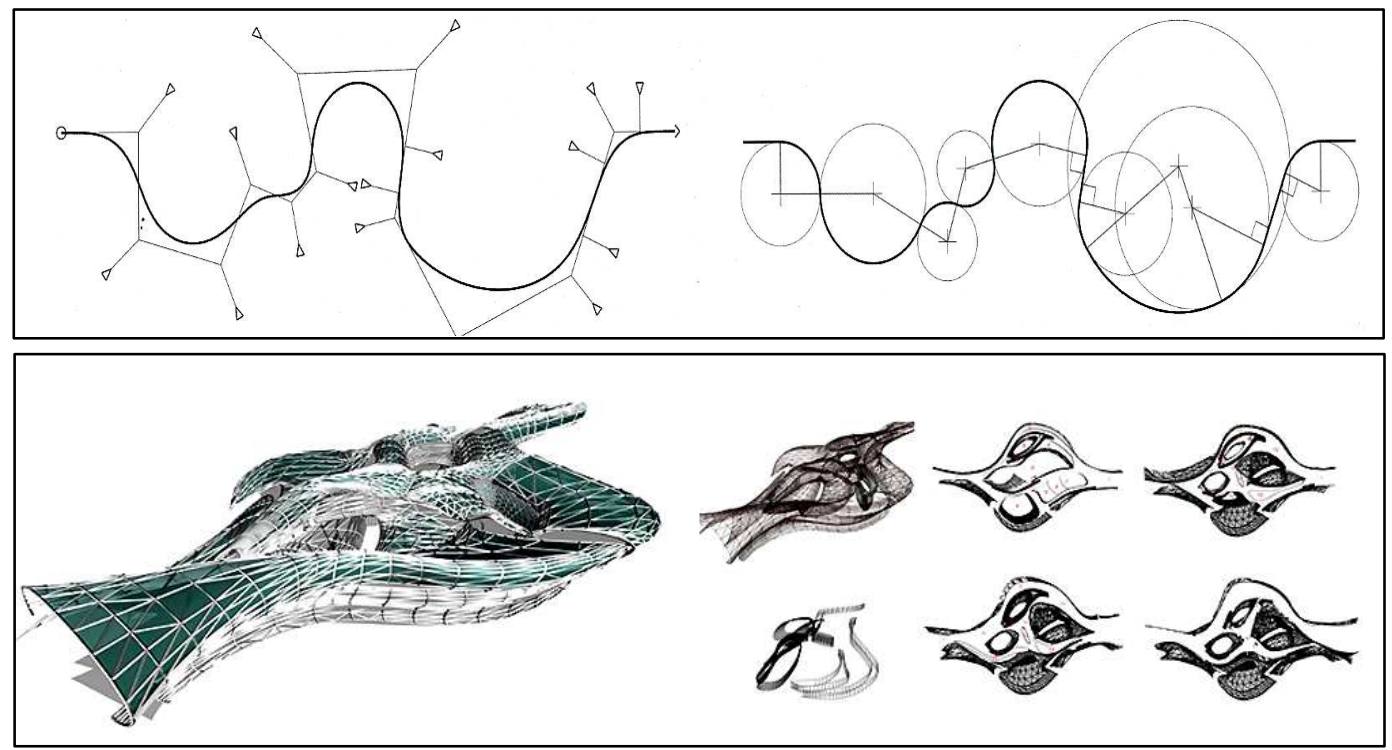

شكل (3) التشكيل بإستخدام التصميم الطبولوجى بالتحكم فى نوعية الخطوط NURBS .
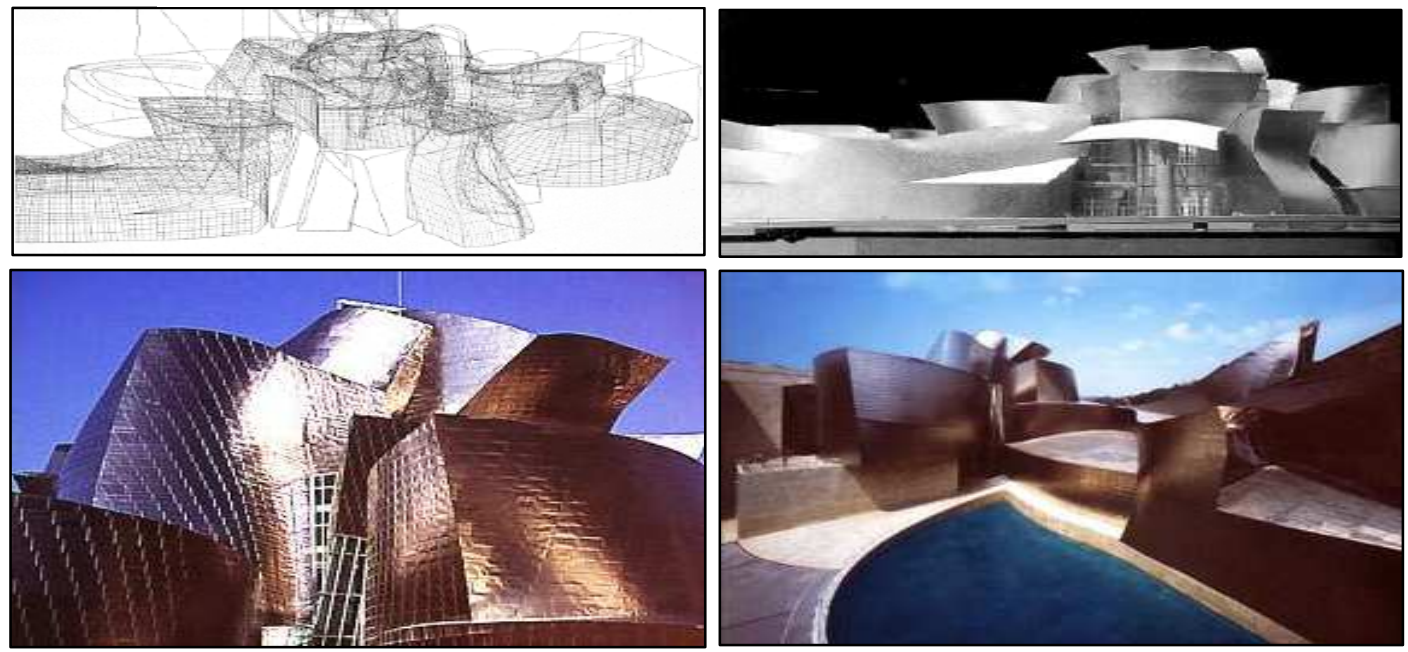

شكل(4) تطبيق للعمارة الطبولوجية فى متحف جوجنهايم "بلباو -اسبانبا" للمعمارى فر انك جيرى كتطبيق للتحكم فى الثبكات.

2-3 Isomorphic architecture ( Isomorphic

3-2 3 العمارة الدتناغهة (الفقاعات): surfaces)

بدأ هذا الاتجاه في التصميم بإستخدام الاشكال المعقدة complicated أو الاشكال ذات الصفة البللورية النقاط

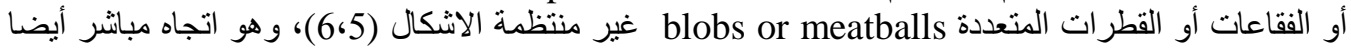

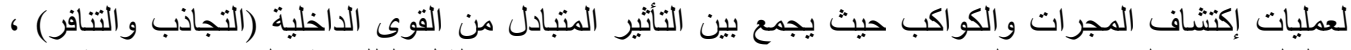

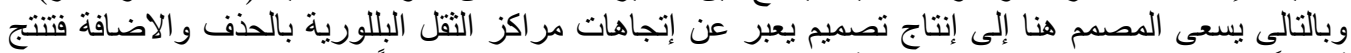

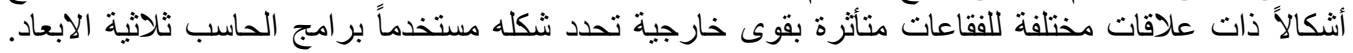

.(Franken, 2002) 

1882
أ.مهد. محمد وهبة إبراهيم
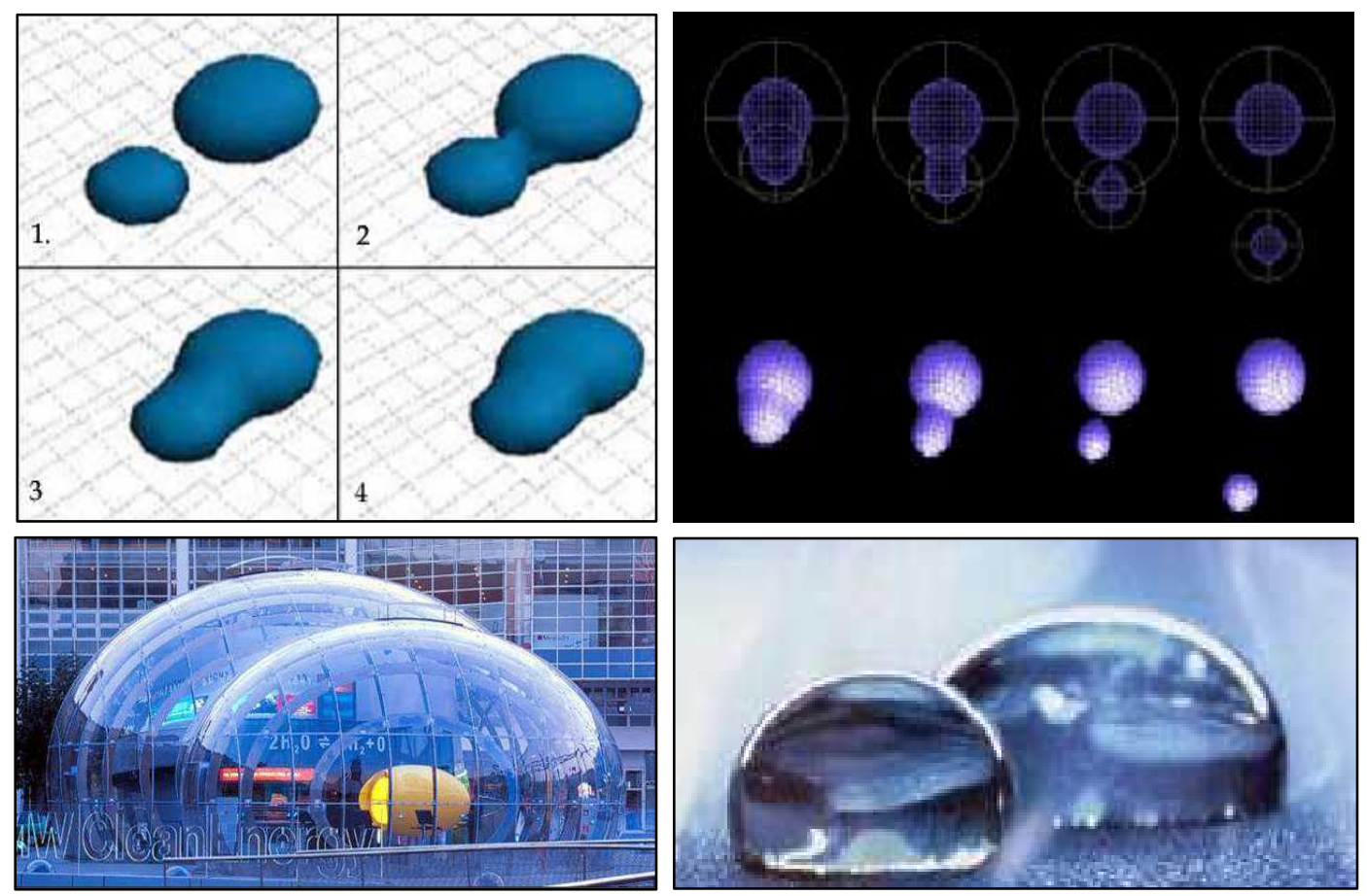

شكل (5) التفكير بالحذف و الاضافة فى التصميم المتناغم بالفقاعاتForms follows forces الثنكل يتبع القوى المؤثرة عليه .
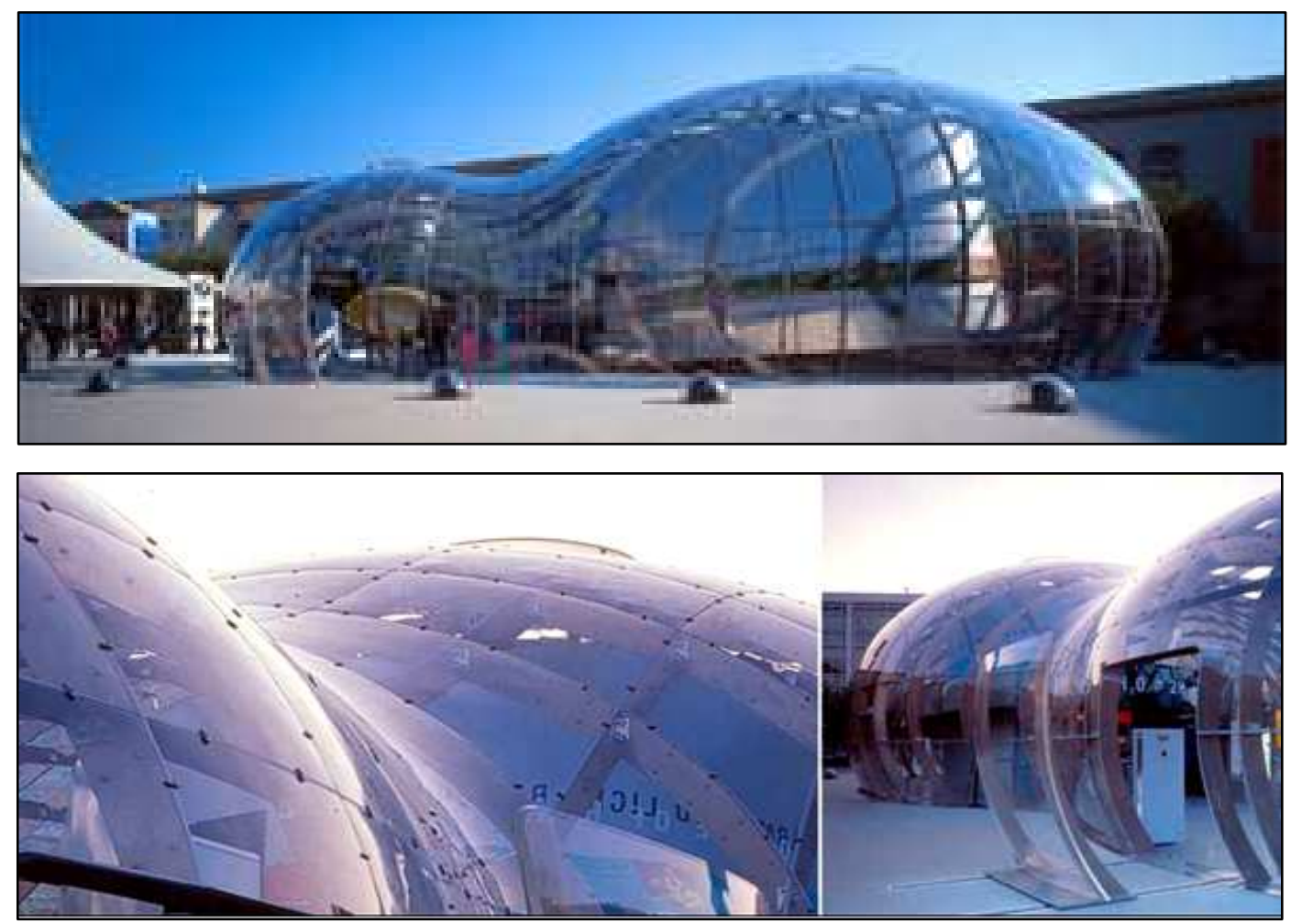

شكل (6) تطبيقات العمارة المتناغمة لمعرض BMW Pavilion فر انكفورت ـ المانيا ـ للمعمارى Bernhard Franken . 


\section{2-4 Animate architecture (Motion kinematics \& العدارة الدتغيرة(الدتحركن) 2-2 dynamics)}

وذللك بإنتخدام برامج الحاسب Kinematics الخاصة بالرسوم المتحركة حيث يتم عمل عدة عمليات لتعديل

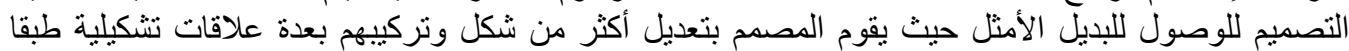

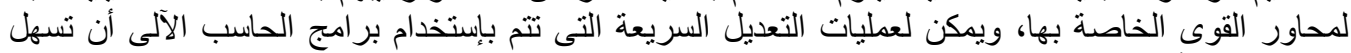

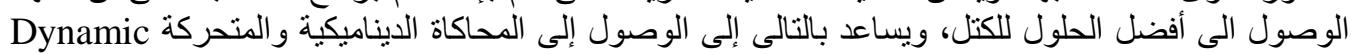
Simulation

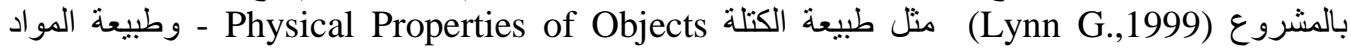

المستخذمة وملائمتها للظروف البيئية المحيطة. شكل(7)
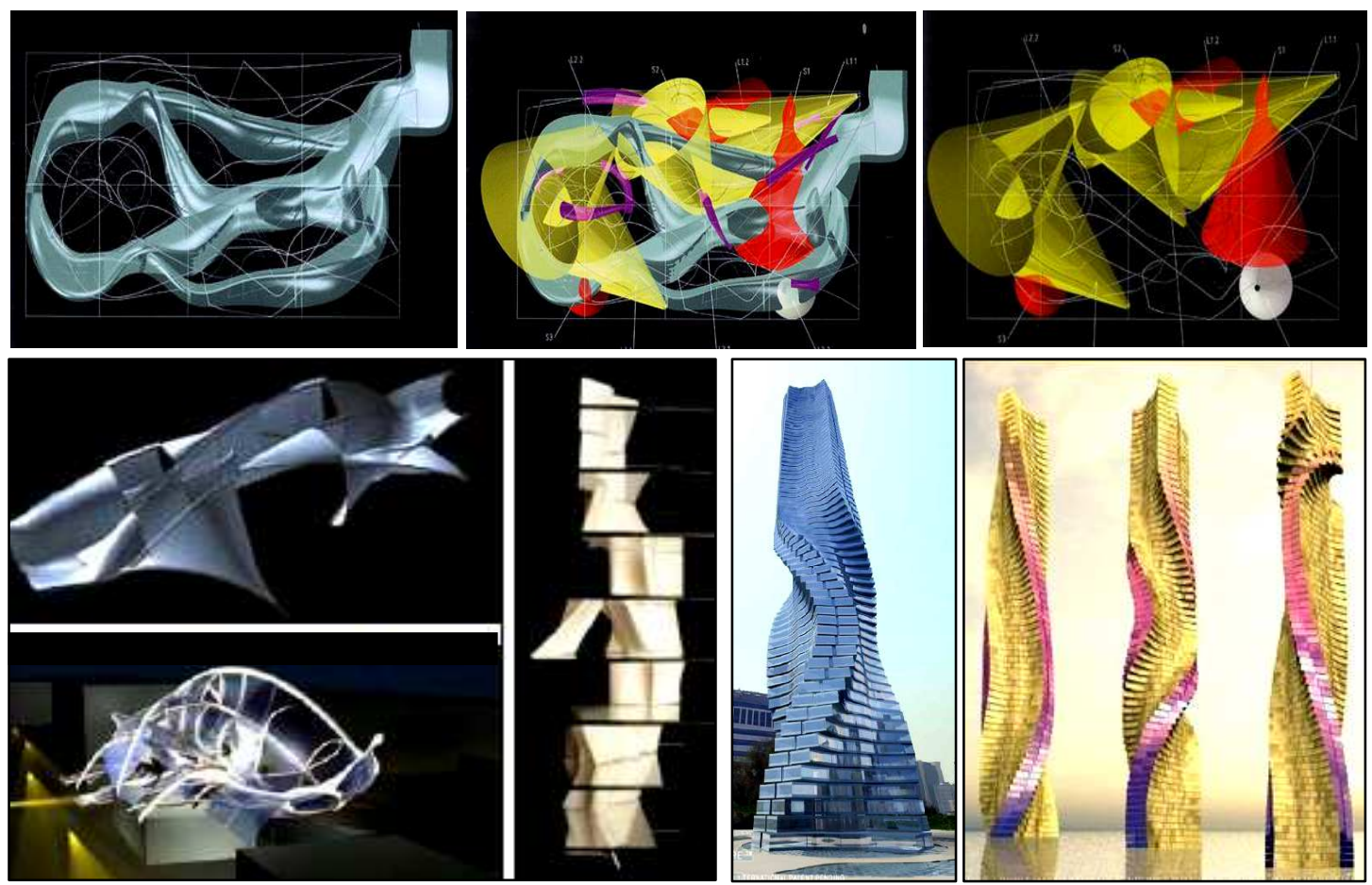

شكل (7 ) العمارة الديناميكية كتطبيق للعمارة المتحركة و المتغيرة .

2- 5 Metamorphic architecture (Key shape animation)

5-2 العدارة الدتحولتئ.

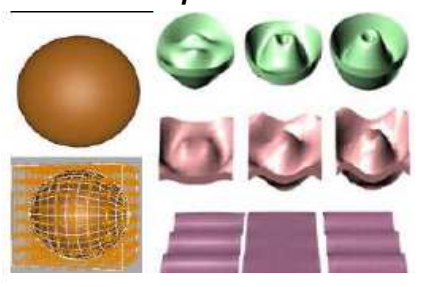

شكل(8) تسجيل التغير فى الاشكال

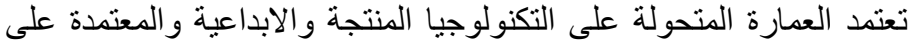

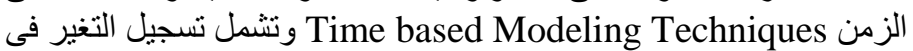
الاشكال و الحجم و اتجاه الثكل و غير ها من الخصائص من خلال

زمن محدد وهو ما يطلق عليه

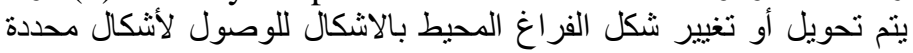

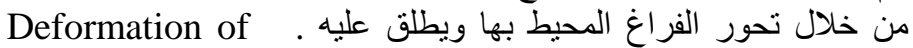
(Lynn G.,1999).(9) modeling space 
2- 6 parametric architecture (Parametric

6-2 6-2 العمارة البار/مترينة، شكل(10) design)
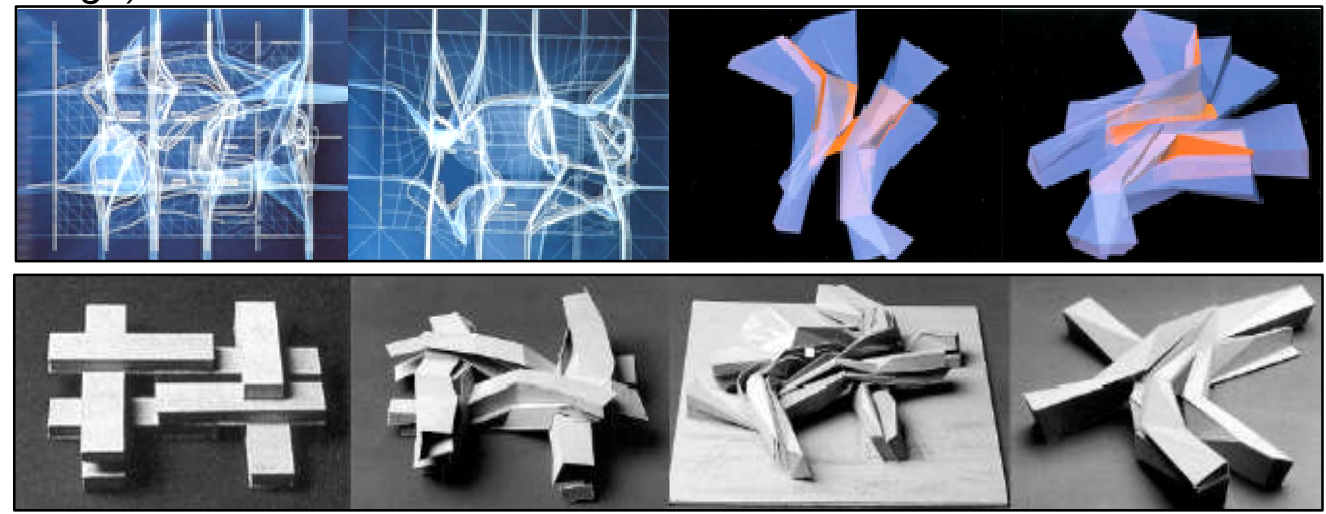

شُكل (9 ) تطبيق للعمارة المتحولة لمبنى شركة BFL للبرمجيات بالهند للمعمارى بيتر ايزمان 1996

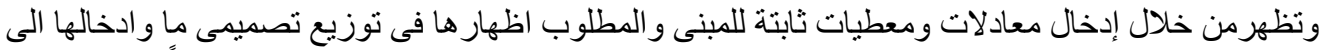

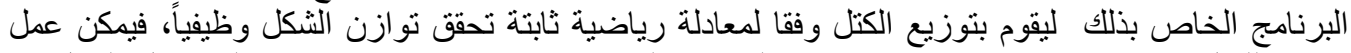

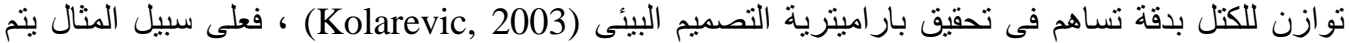

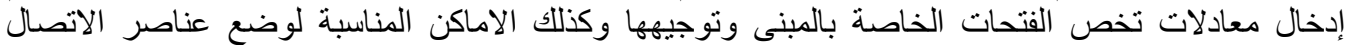

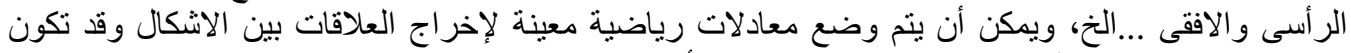

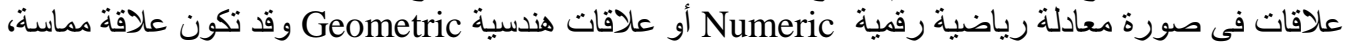

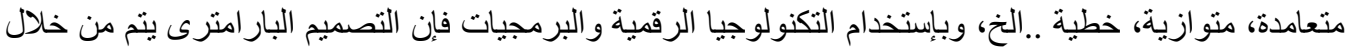

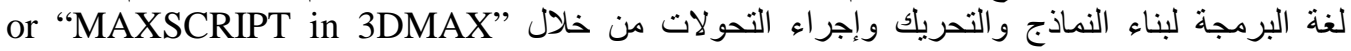
(Rizzoli, 1995). "MEL in MAYA"
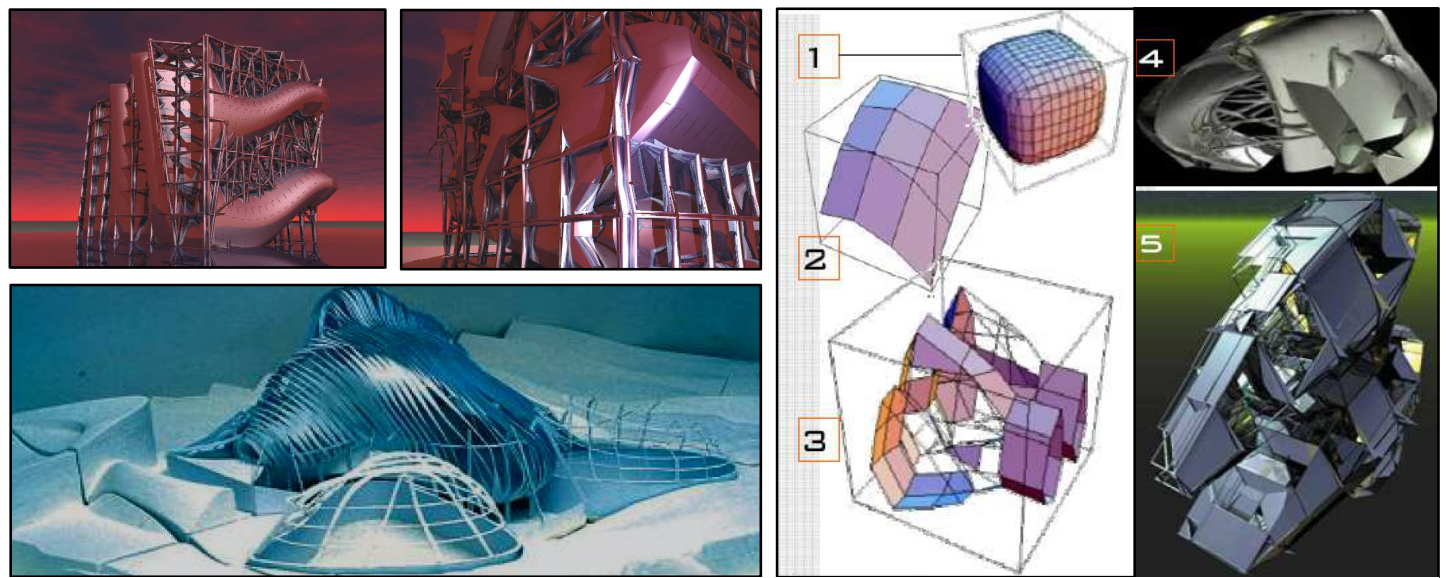

شكل( 10) تطبيقات العمارة البارمنرية بإستخدام البرمجيات و المعطيات المختلفة لبر امج الحاسب الآلى. 
تأثير الجيل الثالث للثورة الرقمية

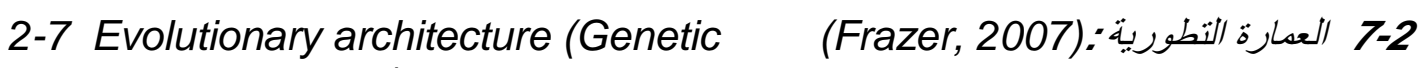
algorithms)

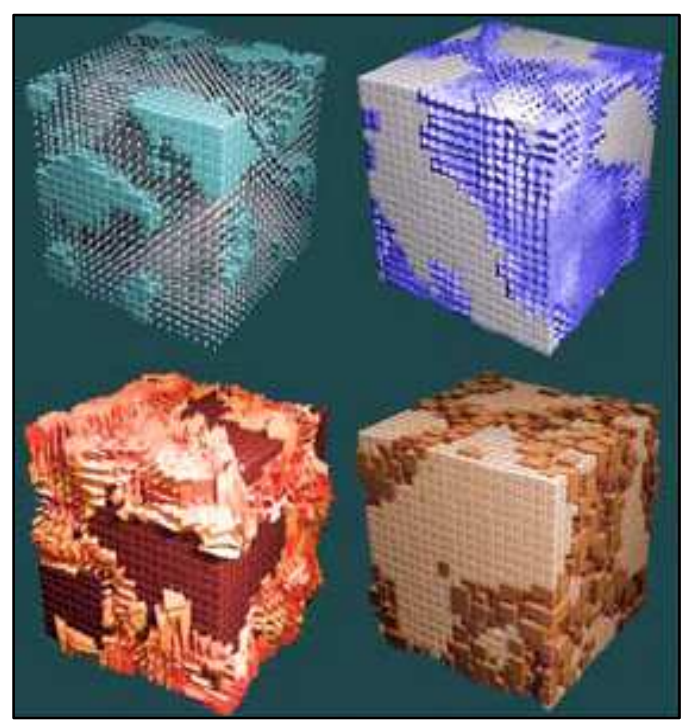

تشكل (11) العمارة التطورية من خلال الاسطح المتفاعلة.
تخضع هنا عملية تطوير الفكرة المعمارية بعمل نموذج

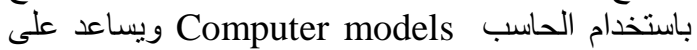
تطوير الفكرة بطرح و اضافة النواه الرئيسية للفكرة،

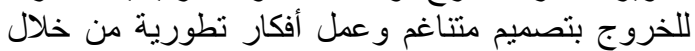

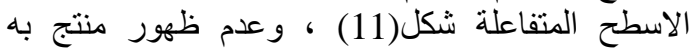

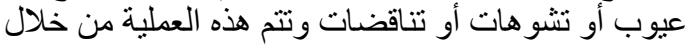

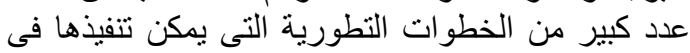

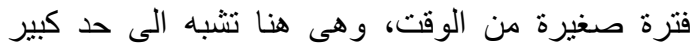

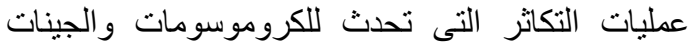

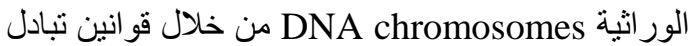
الجينات شكل(12)، وبالتالى فالاسـاس الفكرى للعمارة

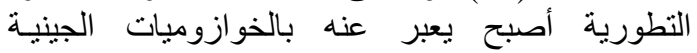

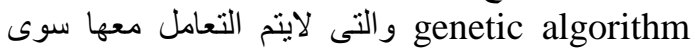
بير امج الكومبيوترشكل(13). (Kolarevic,2011)
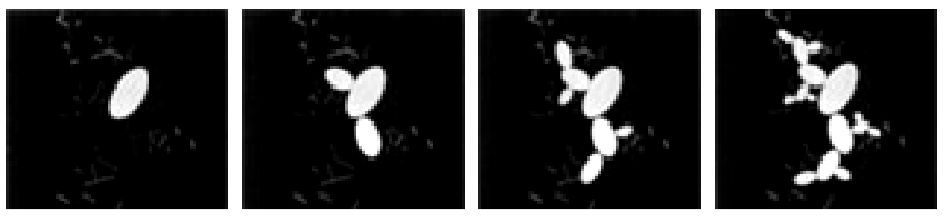

شكل(12) العمارة التطورية ونتابهها مع قو انين التو الد فى الكوموسومات.

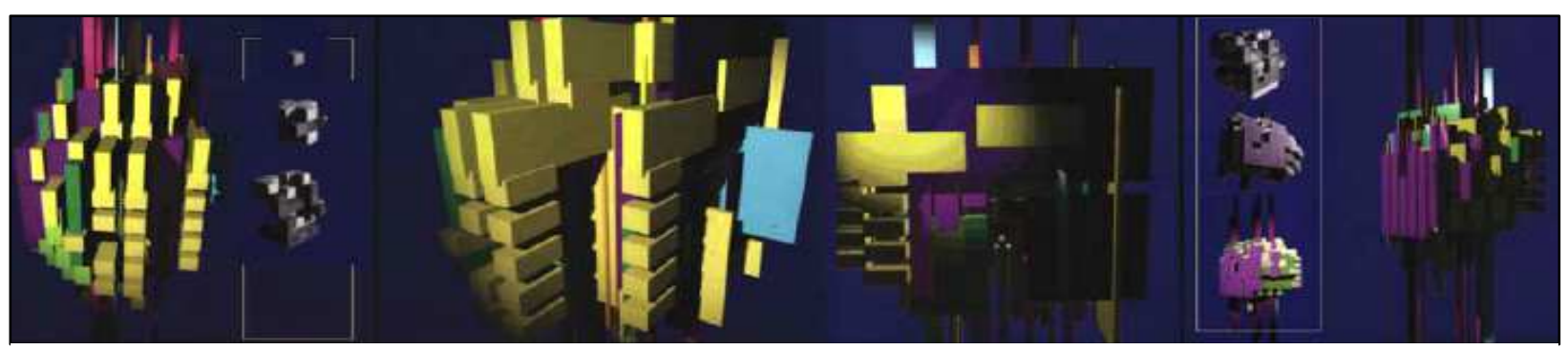

شكل (13) تطبيقات العمارة التطورية بمشروع Generative Sequence للمعمارى Rastogi

The Main Concept for Forming

Computer Usage

Environmental Reference

Geometry Reference

$$
\begin{aligned}
& \text { معايير وهى : جدول (1) (الباحث) } \\
& \text { ـ مفهوم ماور اء الفكرة الرئيسية }
\end{aligned}
$$

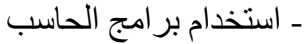

$$
\begin{aligned}
& \text { ـ المرجعية البيئية } \\
& \text { ـ المرجعية المندسية المينية }
\end{aligned}
$$


جدول(1) تصنيف الافكار التصميمية للجيل الثالث الناتجة من الثورة المعلوماتية (الباحث).

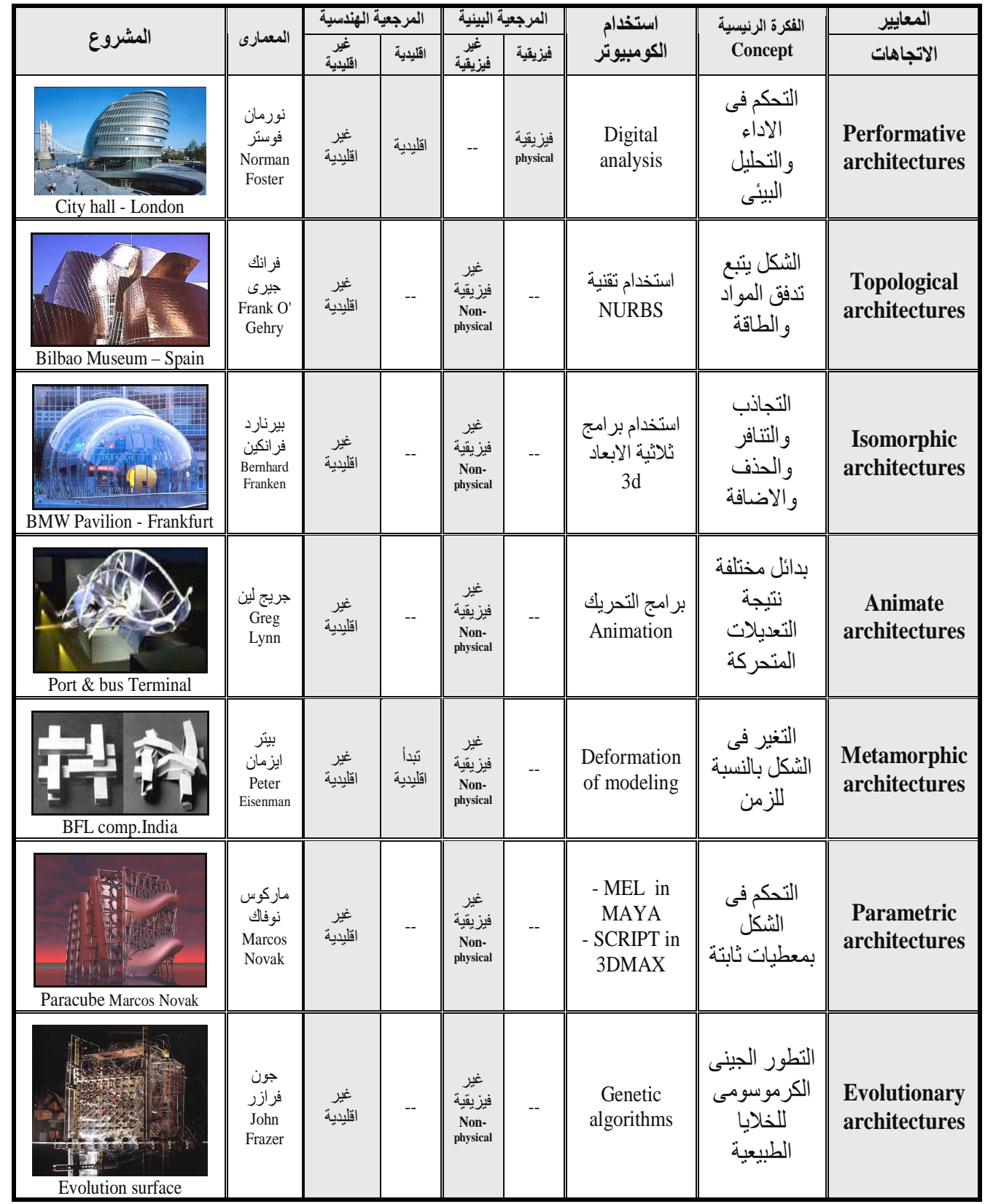

لذللك فإن تطور مراحل التصميم الرقمى هو أمر يتعلق بمدى تفاعل ادوات التصميم المعمارى مع فاعليات

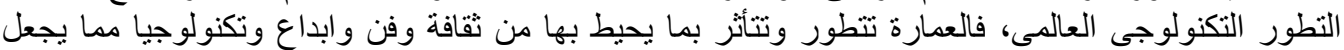

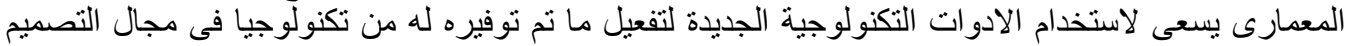

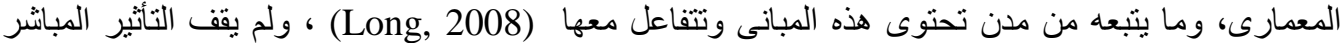

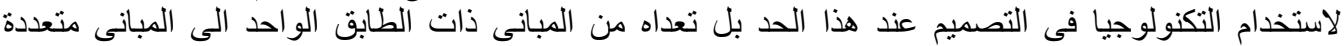

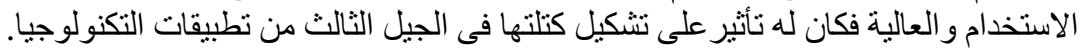




\section{3. المحور الثالث : تثكيل كتل المبانى العالية فى عصر الجيل الثالث للثورة الرقمية}

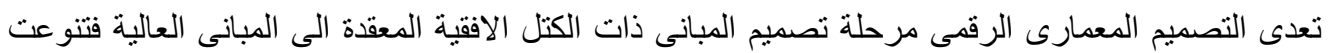

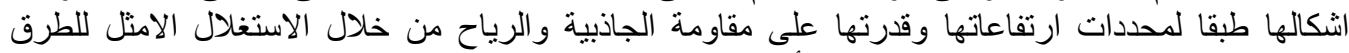

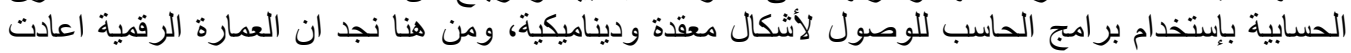

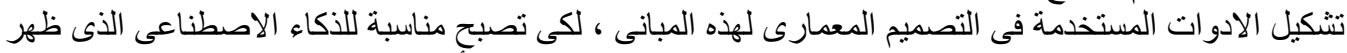

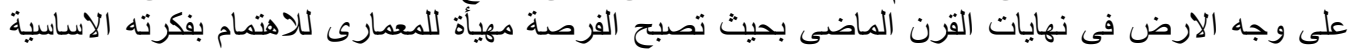

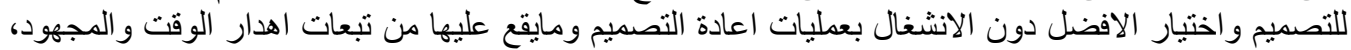

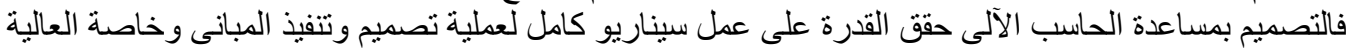

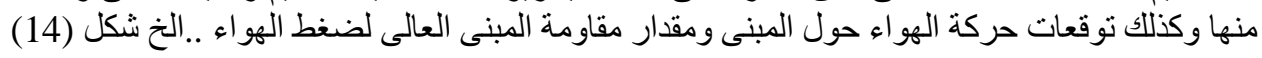

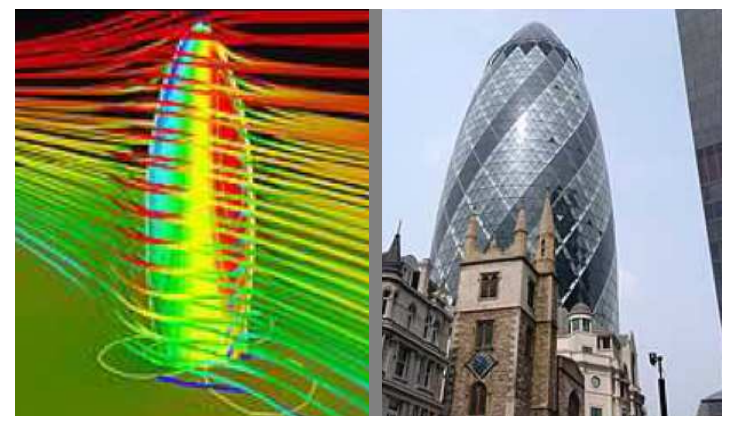

دراسة لتوقعات حركة الرياح حول المبانى العالية قبل التنفيذ

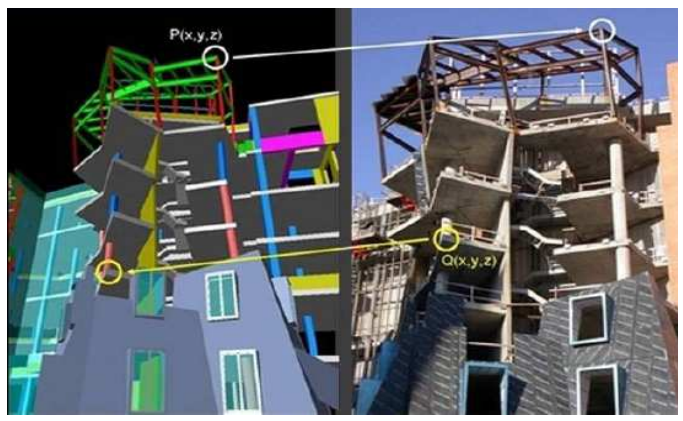

استخدام بر امج الحاسب فى تنفيذ المبانى العالية

\section{شكل(14) تأثثير استخدام الجيل الثالث للتكنولجيا فى تشكيل المبانى العالية .}

ومع الثورة الرقمية ثم المعلوماتية أصبحت المبانى العالية تحتوى العديد من الاستعمالات وبفضل التشابك

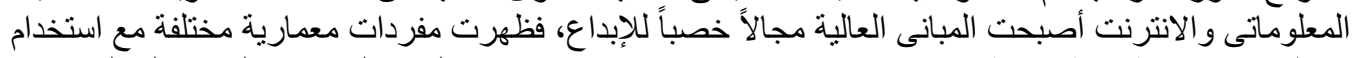

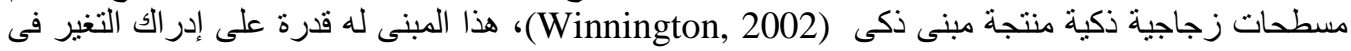

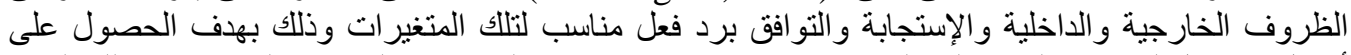

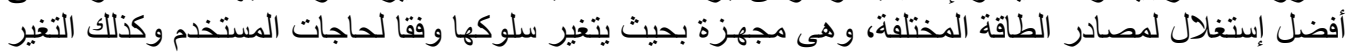

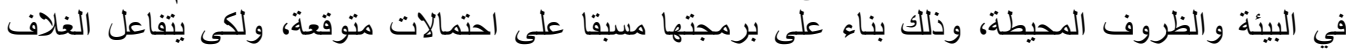

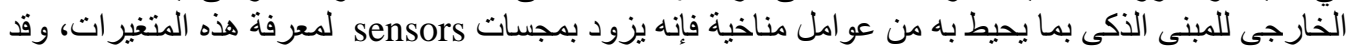

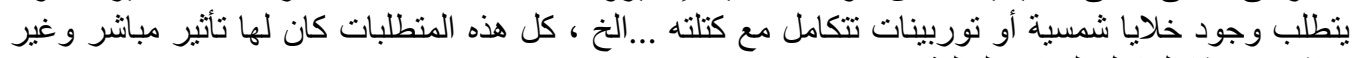

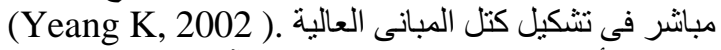
ولقد رأى المعماريون انهم في تحد آخر جديد لقدراتهم الابداعية وفى تتاغم وفكر جديد لإخر اج تشكيلات

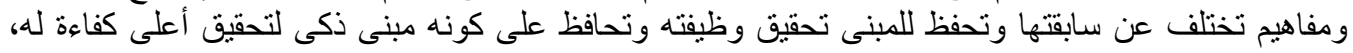
ويمكن تصنيف تشكيل المبانى العالية فى عصر الجيل الثالث للتكنولوجيا (Yeang K, 2002) كما يلى : شكل(15)

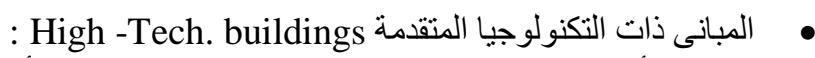

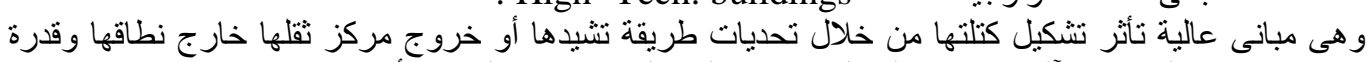

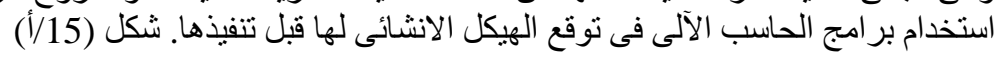

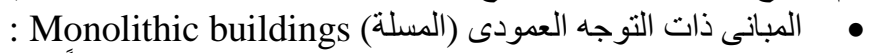

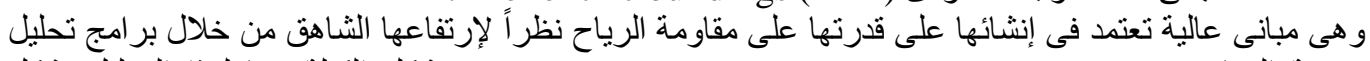

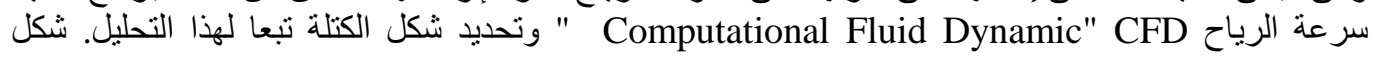




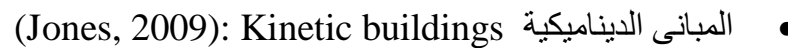

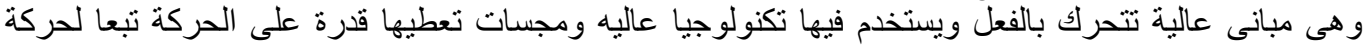

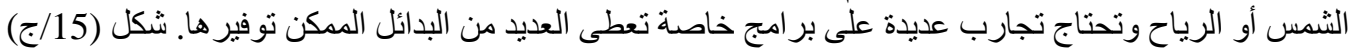

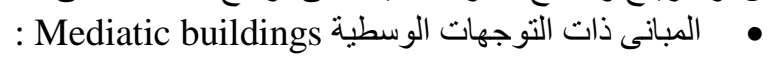

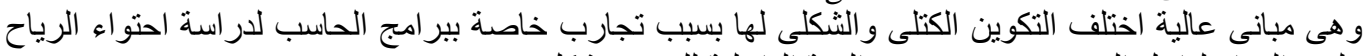

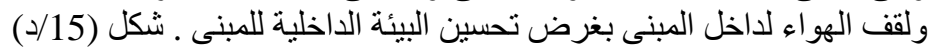

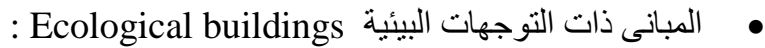

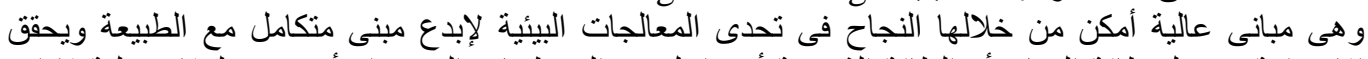
الاستدامة فيستغل طاقة الرياح أو الطاقة الثمسية أويتداخل مع المسطحات الخضر الثراء أو أو يقدم حلو لا مختلفة لإدارة

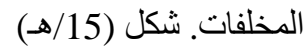

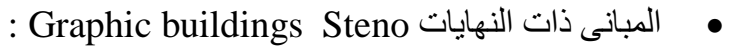

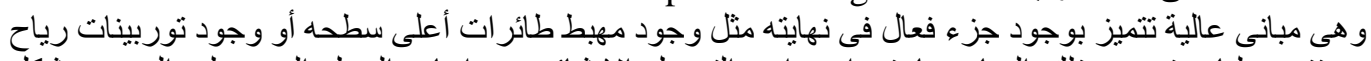
، وتتم عمليات تصميم تلك المبانى بإستخدام بر امج التحميل الانشائى ودراسات الحمل الحمل الحى على المبنى ـ شكل
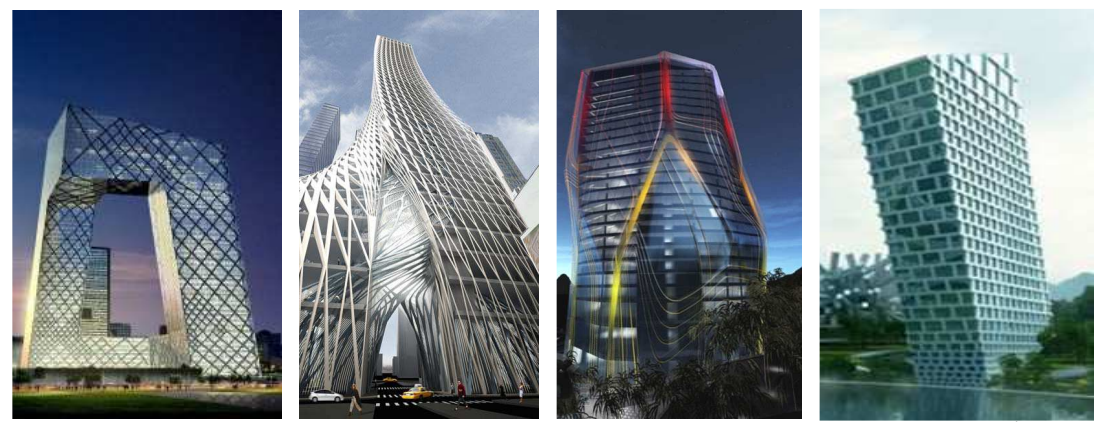

$(g / 15)$

High -Tech. Buildings شكل (15/أ) المبانى العالية ذات التكنولوجيا المتقدمة

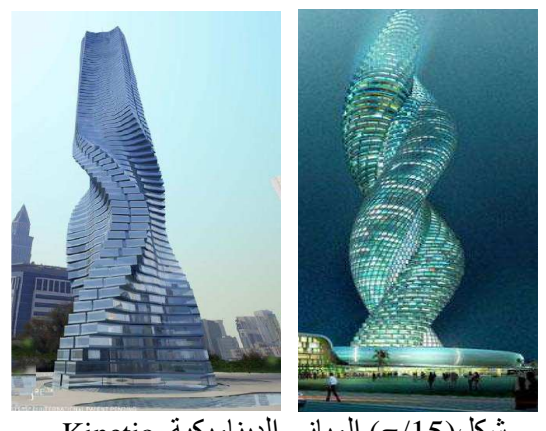

شinetic شكل(15/ج) المبانى الديناميكية buildings
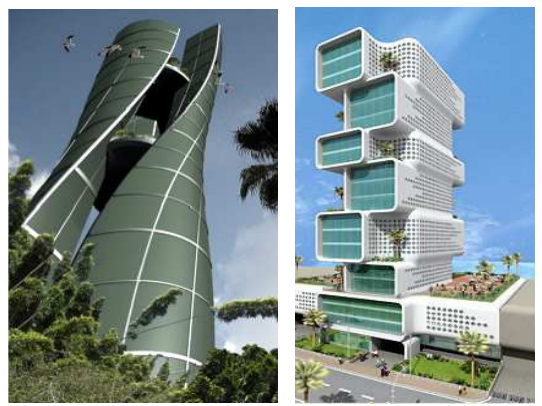

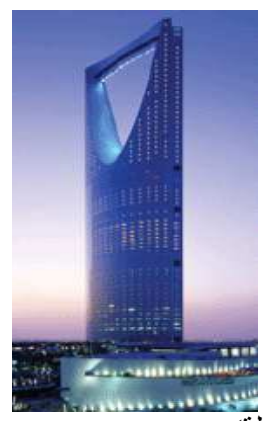

Monolithic شكل (15/ب) المبانى ذات التوجه العمودى (ألمسلة) buildings
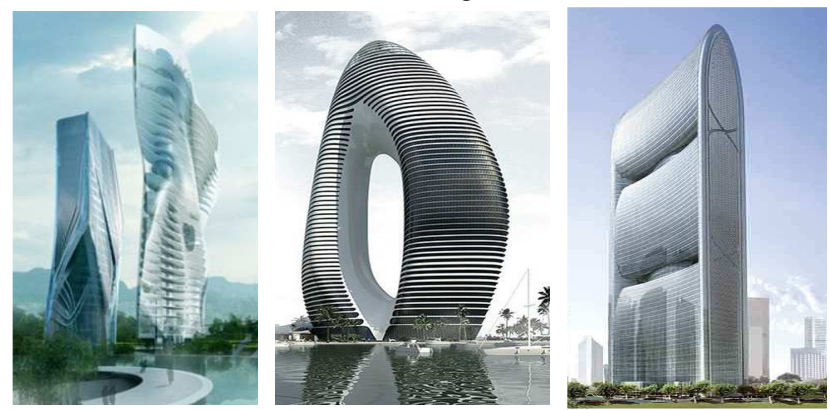
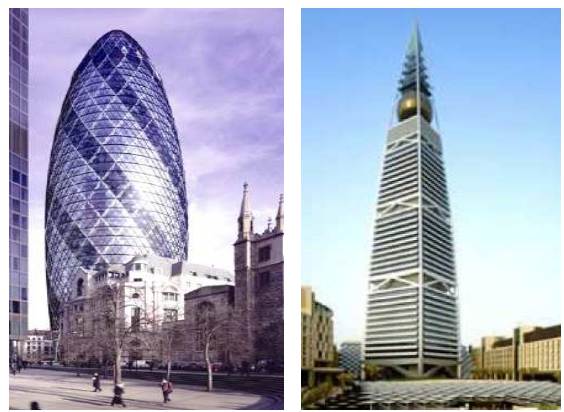

Mediatic buildings شكل(15/د) المبانى ذات التوجهات الوسطية

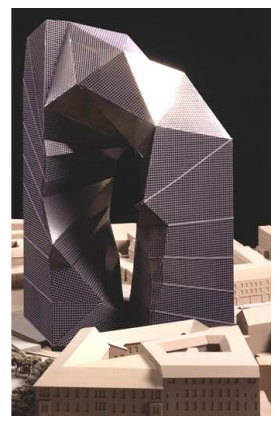
. 

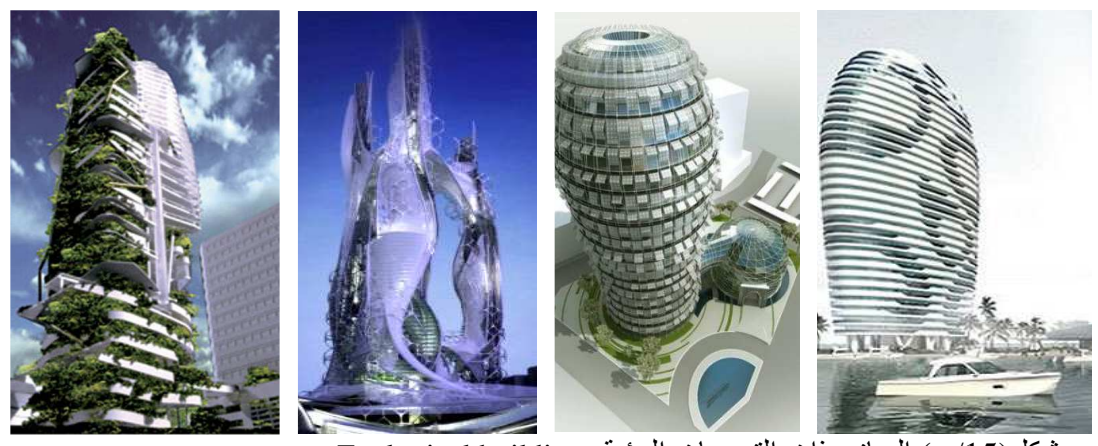

شكل(15/هـ) المبانى ذات التوجهات البيئية Ecological buildings
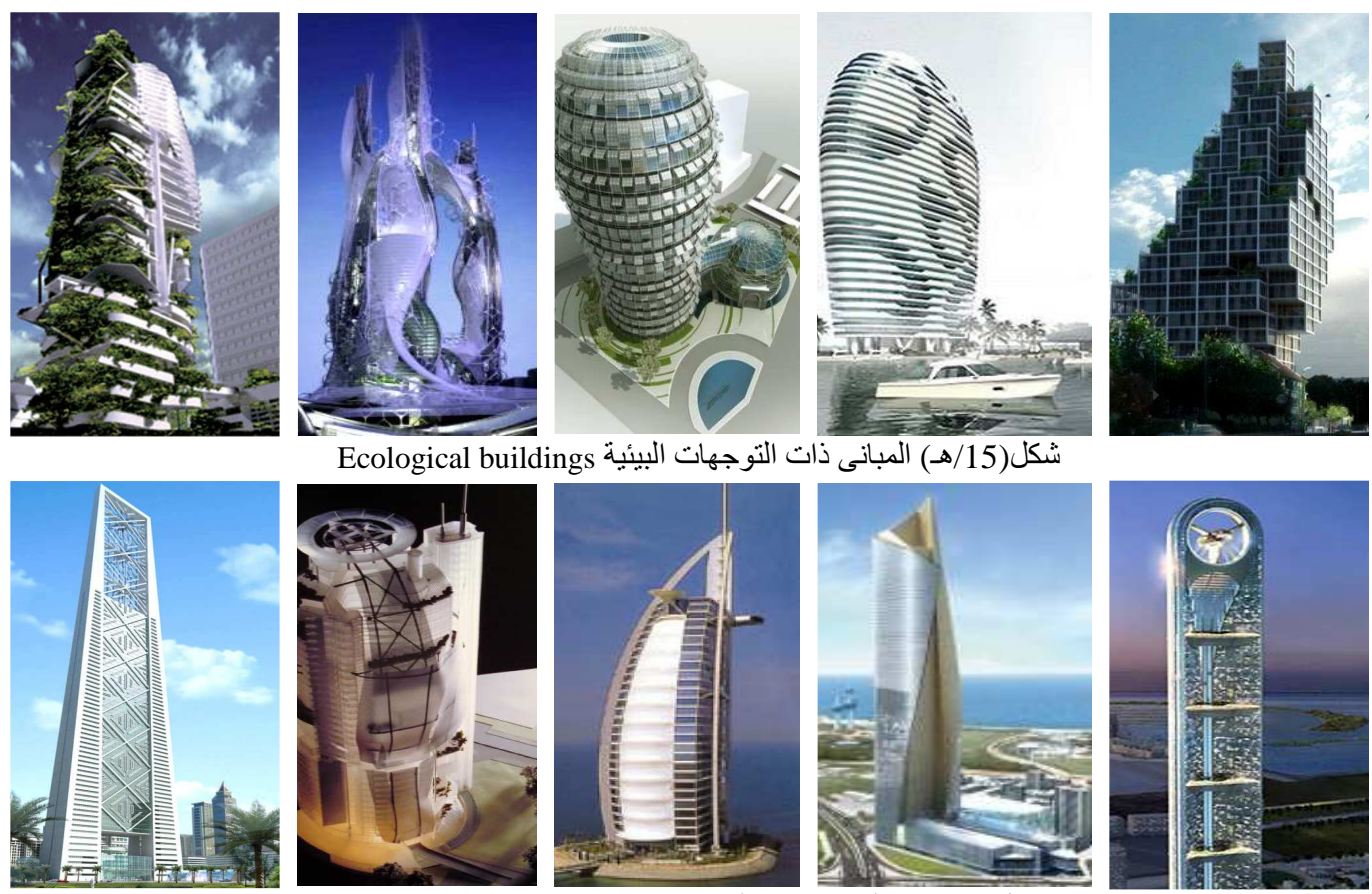

شكل(15/و ) المبانى ذات النهايات Stenographic buildings شكل(15) دور الجيل الثالث للتكنولوجيا فى تنشكيل للمبانى العالية

\section{4 - تأثير برامج الثورة الرقمية على صياغة تثكيل الكتئة المعمارية "الجانب التطبيقى"}

\section{1-4 استبيان وورشة عدل:}

بعد ان تم توضيح الإطار النظري لتونية لتأثير وسائل المعلوماتية في العملية التصميمية وتم التوصل التى صياغة

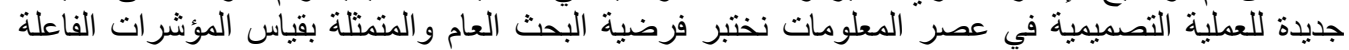

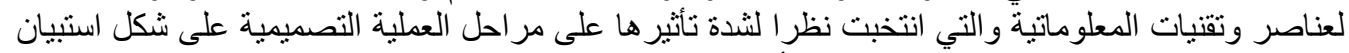

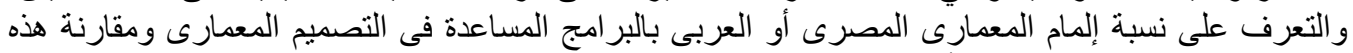

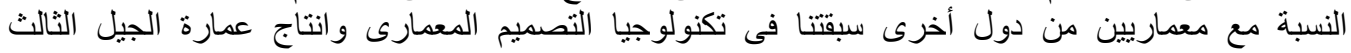
للتكنولوجيا.

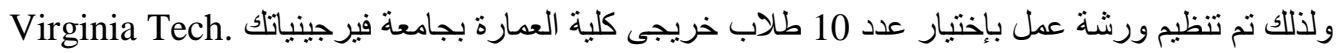

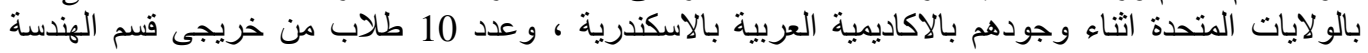

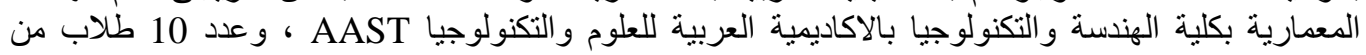

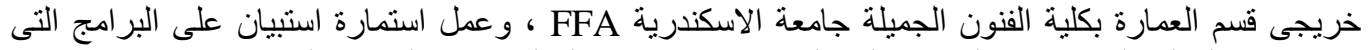

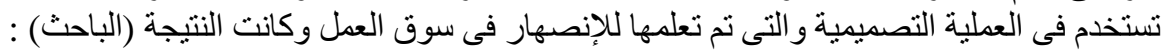


جدول (2) نسب استخدام البرامج المساعدة فى التصميم المعمارى للفئات التعليمية الثلاثة. (الباحث)

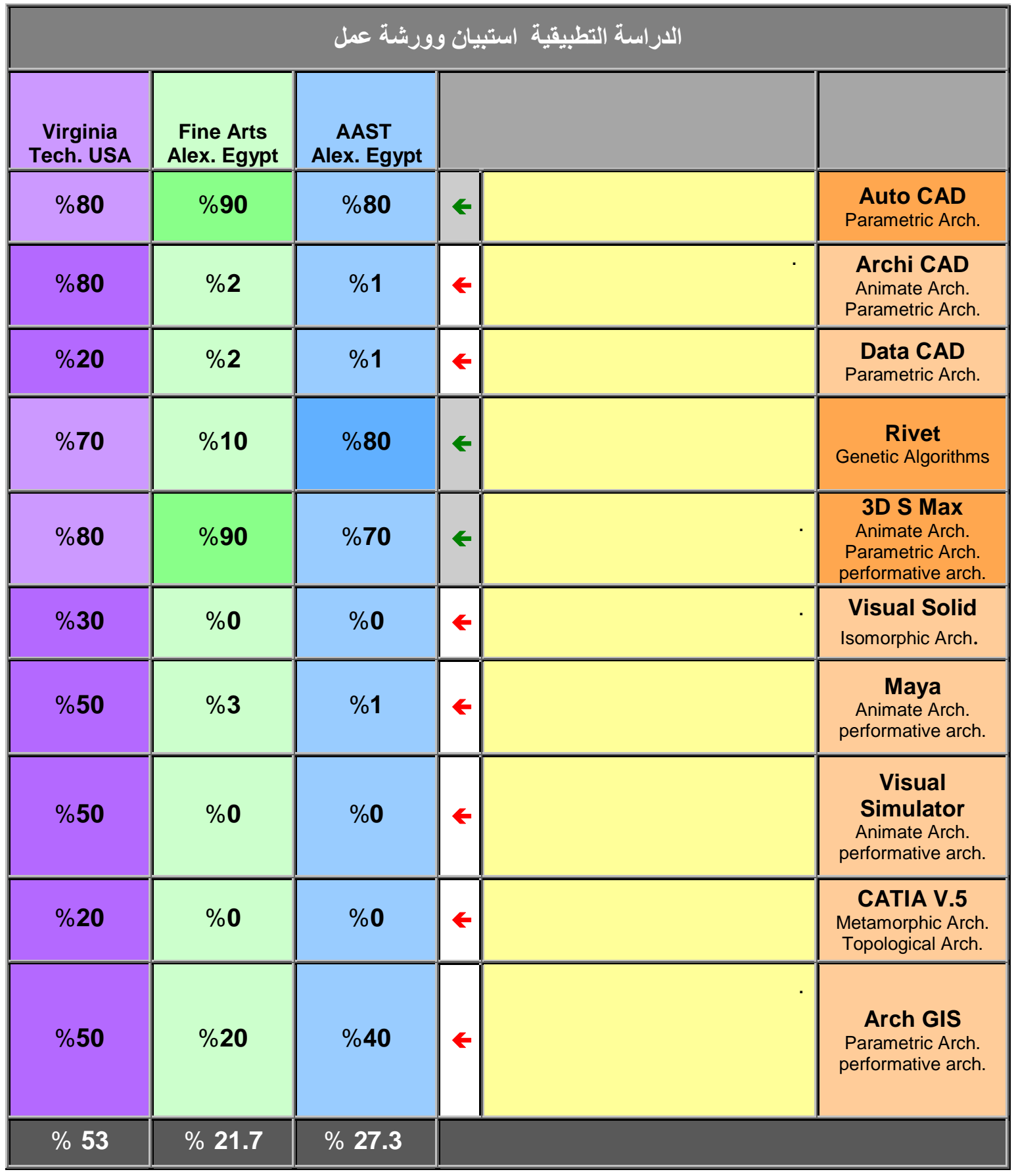


2-4 تحلبل الحالة الدر/سية:

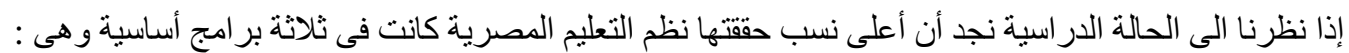

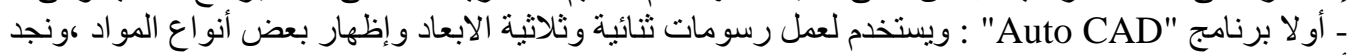

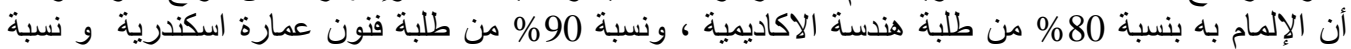

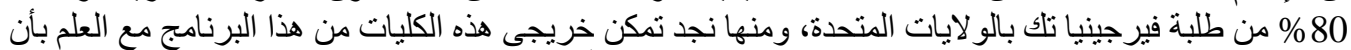

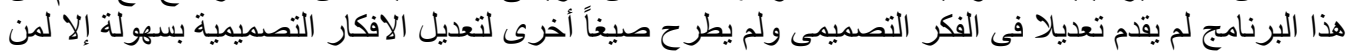

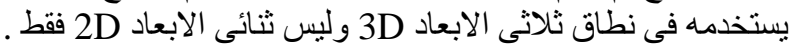

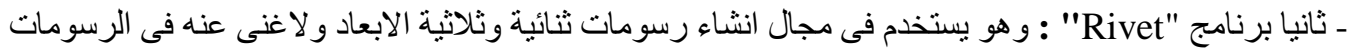

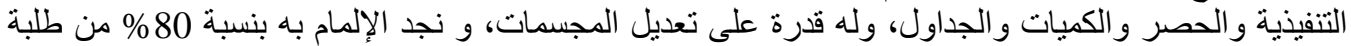

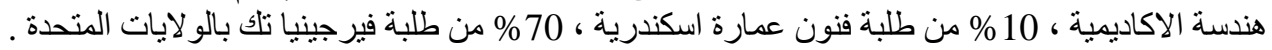

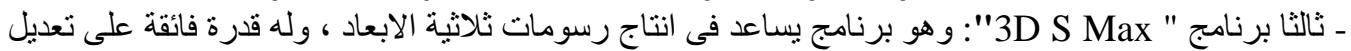

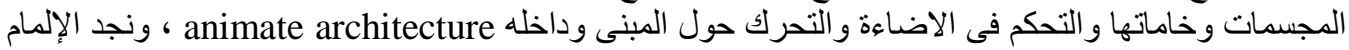

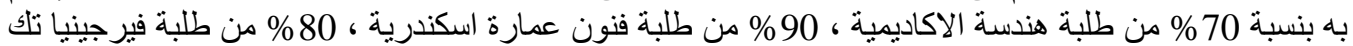

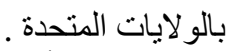

- رابعا بر امج أخرى (Visual Simulator ،Maya ، Visual Solid،Data CAD ،Archi CAD) : (CATIA و هى بر امج تقوم بتقديم نماذج عالية الجودة من المعلومات حول تصميم المشروع ونطاقه وجدوله الزمنى و التكلفة

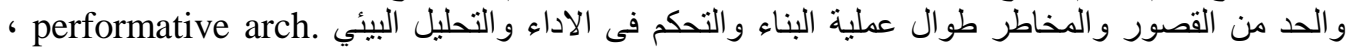

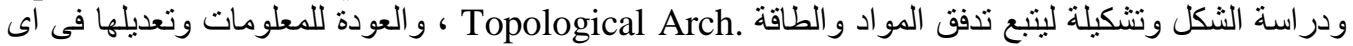

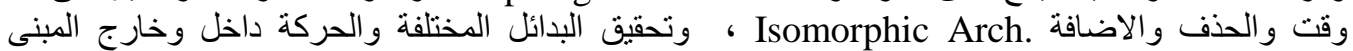

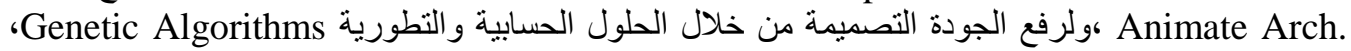

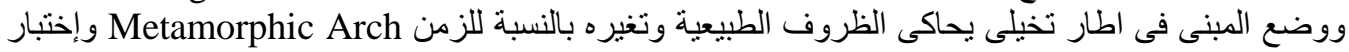

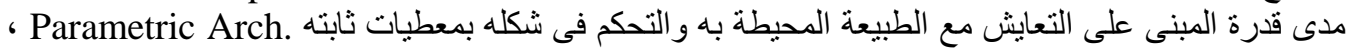

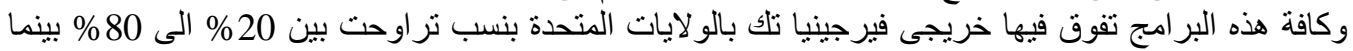
تراوحت نسبة معرفة هذه البرامج لطلبة هندسة الاكاديمية وطلبة فنون عمارة اسكندرية بين 0\%

(الباحث:

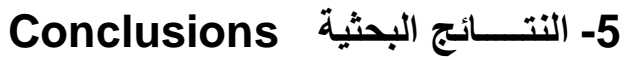

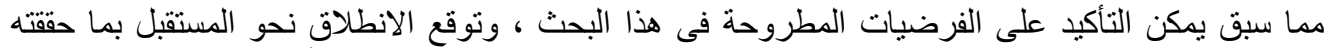

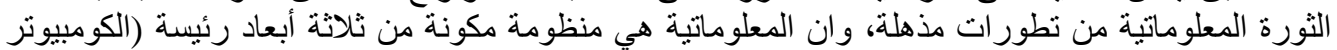

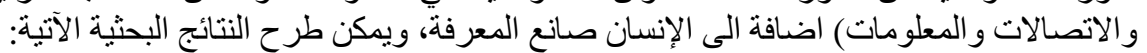

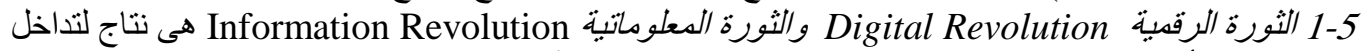

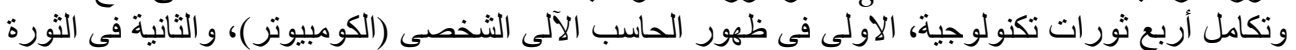

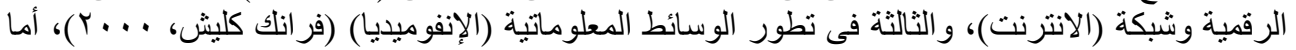

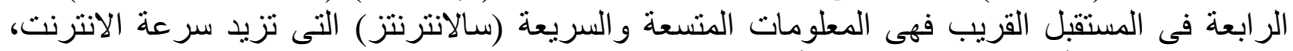

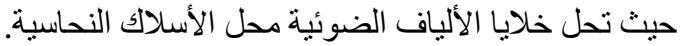

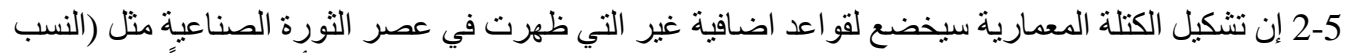

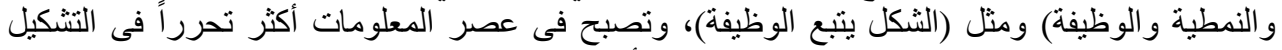

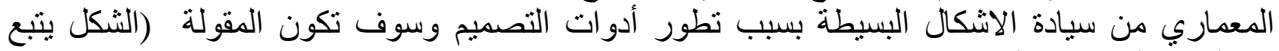
تكنولوجيا التصميم و التنفيذ).

3-5 التصميم المعماري في عصر المعلوماتومات هو عملية منهجية تعتمد في مفرداتها على توظيف ما جلبته لنا

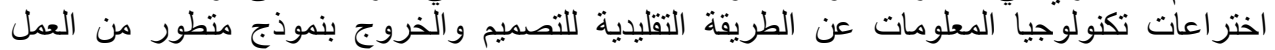

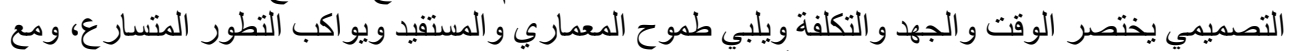

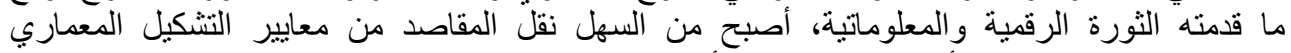

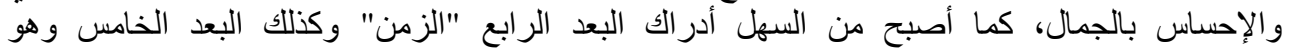


"المعلوماتية" وتأمل التصميمات بمساعدة الحاسب الآلي كأداة تصميمية و تعليمية وبتكاليف تكاد تكون منعدمة. 5-4 شهدت مجالات التشكيل المعماري مولد مصطلحات جديدة لأدوات التصميم الفراغي فاستحثثت البرامج

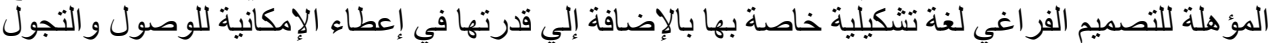

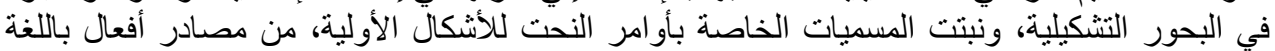

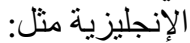
(Twist, Loft, Extrude, Revolve, and Mesh editing)

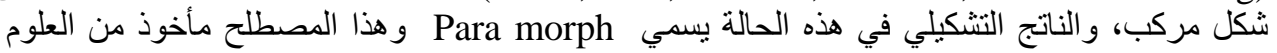
الجيولوجية ويطلق علي الفلزات التي تحيد عن سيرتها كتعبير عن تحول الكتلة من الشكل البسيط الى لى الى

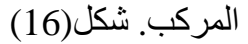

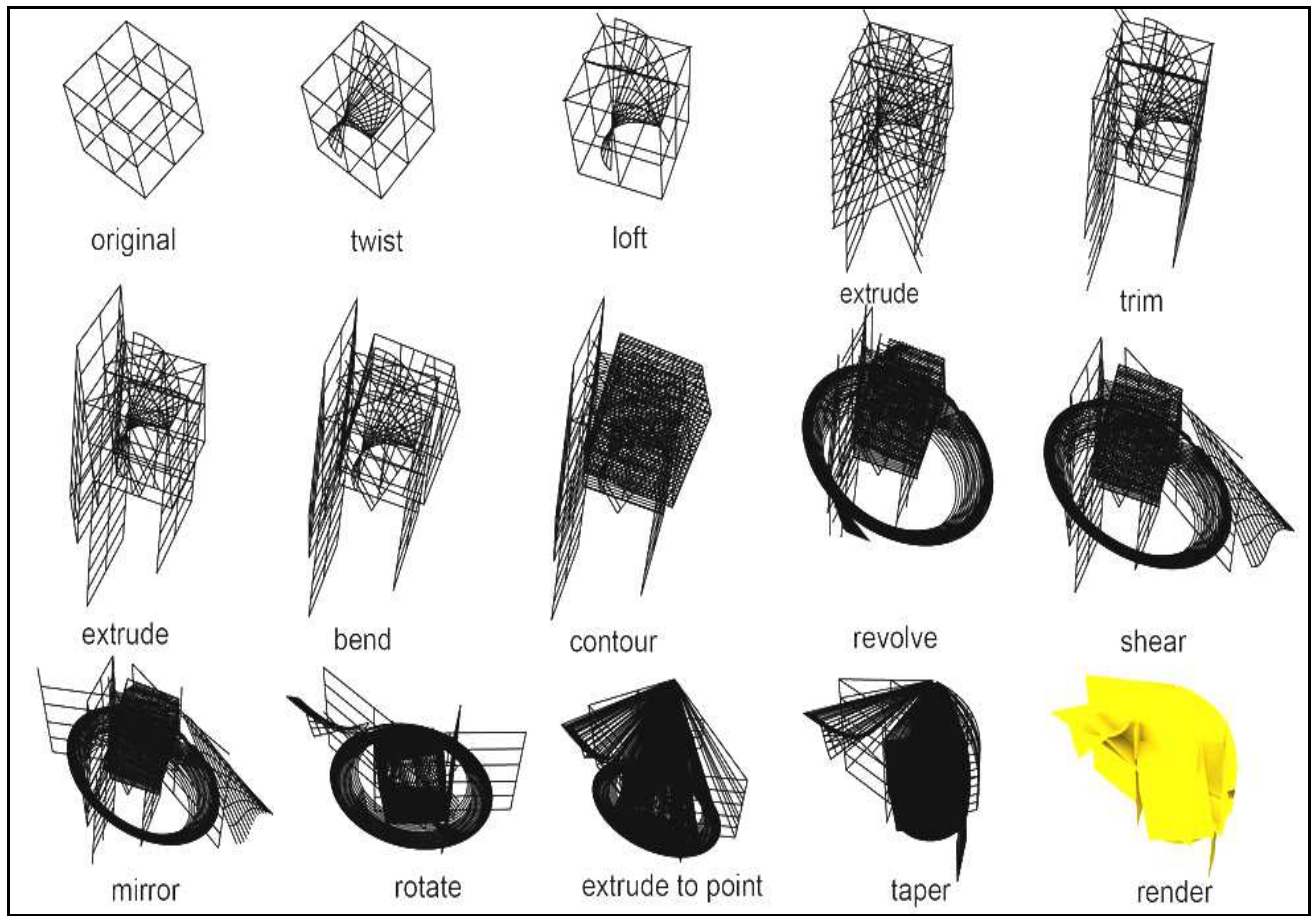

شكل (16) التعبير التشكيلي Para morph باستخدام برامج الحاسب وتطور الأشكال وصو لا للشكل النهائي. (Yoon, Shyan, 2008)

\section{Recommendations 6}

6-1-6 ضرورة التعرف على البعد المعلوماتى فى مادة التصميم المعمارى فى المنهج التعليمى لأقسام الهندسة

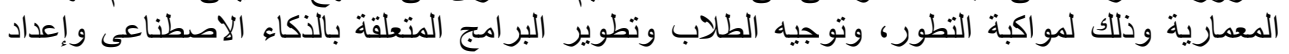
وتتفيذ بر امج تعليمية ودور ات تدريبية لطلاب كليات العمارة ودئ ونهنسي المؤسسات العاملة في مجال التصميم المعمارى.

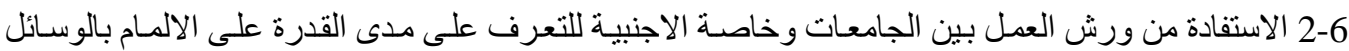

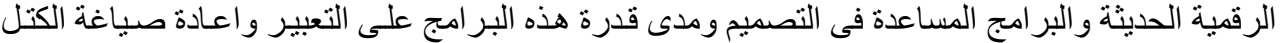

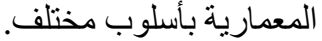


3-6 ضرورة تحول المعماريين من مجرد مستهلكين للتطبيقات المعمارية وتقلبد الغرب فى تشكل الكتل المعمارية

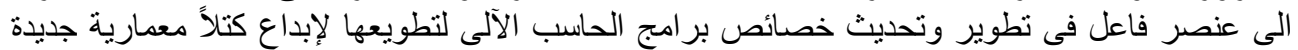

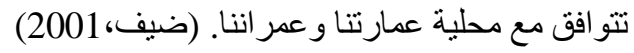

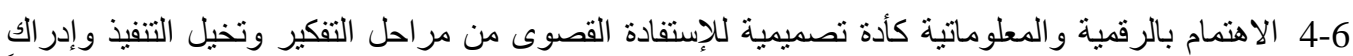

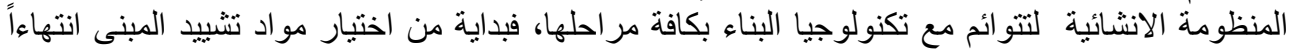
بمواد النهو التشطيب.

6-5 توجيه البحث العلمى وخاصة فى علوم العمارة والتخطيط لتحليل واستنباط التغير ات التفصيلية المتوقع حدوثها

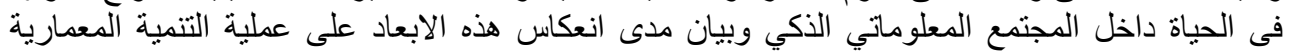

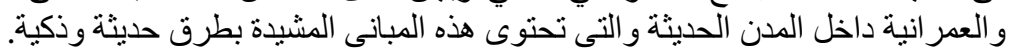

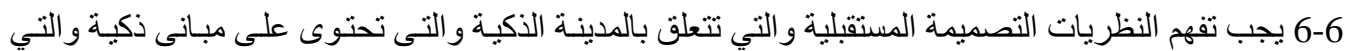

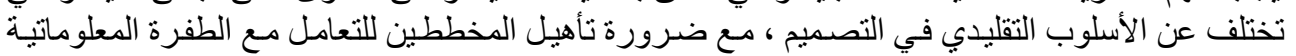

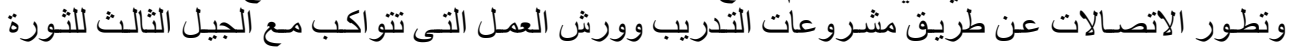
الرقمية.

\section{7- المراجع References}

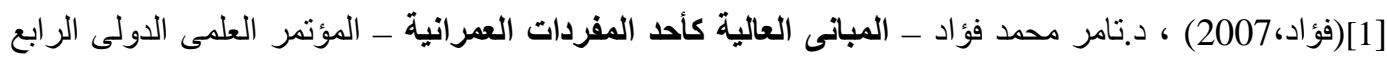

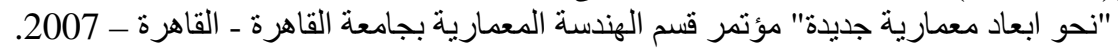

[2] (ضيف،2001) ، محمد ضيف "تخطيط المدينة العربية فى الالفية الثالثة.. نحو اعادة صياغة المعايير

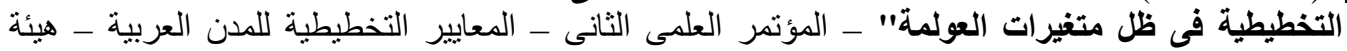
المعماريين العرب ، طر ابلس ، الجماهيرات العولمة" العربية العربية الليبية ، 2001.

[3] ( فر انك كليش ، . . . . ) ـ فر انك كليش "تورة الإنفوميديا ، الوسائط المعلوماتية وكيف تغير عالمناوحياتكا"

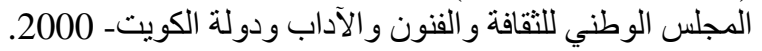

[4] (Betsky ,2010) Aaron "New Architecture in the Emerging World" Thames\& Hudson Ltd, High Holborn, London, Uk, 2010.

[5] (Kolarevic,2000), Branko Kolarevic,'Digital Architectures" in M.Clayton and G.Velasco(eds.), Proceedings of the ACADIA 2000 Conference, ACADIA.2000.

[6] (Kolarevic,2001), Branko Kolarevic, "Designing and Manufacturing Architecture in the Digital Age", Proceedings of the ACADIA, 2001 Conference, ACADIA

[7] (Kolarevic, 2003) , Branko Kolarevic “Architecture with Digital Design”, Taylor and Francis Pub., New York .2003

[8] (Kolarevic,2011) Branko Kolarevic ,'Digital Morphogenesis and Computational Architectures" Lecture, Available online : http://www.gsfa .upenn.edu/arch742/lectures/lecture6.html 2011.

[9] (DDC,1999), Department of design and construction (DDC) "High Performance Building Guidelines” City of New York Publications, New York, 1999.

[10] (Franken,2002), Bernhard Franken "Bernhard Franken work Franken Architekten" Frankfurt, Germany,2002. 
[11] (Frazer, 2007) John Frazer "An Evolutionary Architecture" Architectural Association ,London,UK 2007.

[12] (Jencks, 2000), Charles Jencks, "Metabolism in Architecture "Theories \&

Manifestoes of Contemporary Architecture - Kisho Kuro Kawa",Academy Edition , London UK, 2000

[13] (Jones,2009) Will Jones "Un built Masterworks of the $21^{\text {st }}$ Century Inspirational Architecture for the Digital Age", Thames\& Hudson Ltd , High Holborn, London, Uk, 2009 .

[14] (Long,2008) Kieran Long “The New Architectural Generation” Laurence King Publishing Ltd, London, UK, 2008.

[15] (Lynn ,G.,1999) Greg Lynn in collaboration with Michael McInturf and Douglas Garofalo “Animate Form” Princeton Architectural Press, New York, 1999.

[16] (Peterson 1990), George E Peterson,. , " Multi- sectoral Investment planning, " Urban Management Program" Discussion paper - prepared for UNCHS (Habitat),1990.

[17] (Rizzoli. 1995) Rizzoli Mitchell, W. and M. Mc.Cullough.“ Hybrid space: new forms in digital architectures". New York, USA, 1995.

[18] ( United Nations, 1996), United Nations Conference On Human Settlements, Habitat-II, The Habitat Agenda: Global plan of Action. Istanbul, 1996.

[19] (Winnington ,M. \& Harris,J. 2002.), "Intelligent Skins" , Architectural Press , Oxford , 2002.

[20] (Yeang, 1996) ken Yeang,"The Skyscraper Bioclimatically Considered”, Academy Group , London ,1996.

[21] (Yeang, 2002 ) - ken Yeang, "Reinventing The Skyscraper A Vertical Theory of Urban Design" John Wiley \& Sons Academy Group , London ,2002.

[22] (Yeang K, 2007 ) - Ken Yeang, Ivor Richard , "Eco Skyscraper" Images publishing Group Pty Ltd , Mulgrave, Victoria , Australia ,2007

[23] (Yoon, 2008) Shyan Yoon "Digital Diagram II Architecture+Interior" Jeong Kwang Young, ARCHIWORLD Co.,Ltd, Gwangjin-gu, Seoul, Korea, 2008. 